\title{
A New Algorithm for Euclidean Shortest Paths in the Plane*
}

\author{
Haitao Wang \\ Department of Computer Science \\ Utah State University, Logan, UT 84322, USA \\ haitao.wang@usu.edu
}

\begin{abstract}
Given a set of pairwise disjoint polygonal obstacles in the plane, finding an obstacle-avoiding Euclidean shortest path between two points is a classical problem in computational geometry and has been studied extensively. Previously, Hershberger and Suri [SIAM J. Comput. 1999] gave an algorithm of $O(n \log n)$ time and $O(n \log n)$ space, where $n$ is the total number of vertices of all obstacles. Recently, by modifying Hershberger and Suri's algorithm, Wang [SODA 2021] reduced the space to $O(n)$ while the runtime of the algorithm is still $O(n \log n)$. In this paper, we present a new algorithm of $O(n+h \log h)$ time and $O(n)$ space, provided that a triangulation of the free space is given, where $h$ is the number of obstacles. Our algorithm builds a shortest path map for a source point $s$, so that given any query point $t$, the shortest path length from $s$ to $t$ can be computed in $O(\log n)$ time and a shortest $s$ - $t$ path can be produced in additional time linear in the number of edges of the path.
\end{abstract}

\section{CCS CONCEPTS}

- Theory of computation $\rightarrow$ Computational geometry; Design and analysis of algorithms.

\section{KEYWORDS}

shortest path, shortest path map, shortest path query, Euclidean distance, obstacle avoidance, polygonal domain

\section{ACM Reference Format:}

Haitao Wang. 2021. A New Algorithm for Euclidean Shortest Paths in the Plane. In Proceedings of the 53rd Annual ACM SIGACT Symposium on Theory of Computing (STOC '21), June 21-25, 2021, Virtual, Italy. ACM, New York, NY, USA, 14 pages. https://doi.org/10.1145/3406325.3451037

\section{INTRODUCTION}

Let $\mathcal{P}$ be a set of $h$ pairwise disjoint polygonal obstacles with a total of $n$ vertices in the plane. Let $\mathcal{F}$ denote the free space, i.e., the plane minus the interior of the obstacles. Given two points $s$ and $t$ in $\mathcal{F}$, we consider the problem of finding a Euclidean shortest path from $s$ to $t$ in $\mathcal{F}$. This is a classical problem in computational geometry and has been studied extensively, e.g., [3, 18-23, 32, 34, 36, 39, 41, 42, 44].

*This research was supported in part by NSF under Grant CCF-2005323. A full version of this paper is available at https://arxiv.org/abs/2102.12589

Permission to make digital or hard copies of all or part of this work for personal or classroom use is granted without fee provided that copies are not made or distributed for profit or commercial advantage and that copies bear this notice and the full citation on the first page. Copyrights for components of this work owned by others than ACM must be honored. Abstracting with credit is permitted. To copy otherwise, or republish, to post on servers or to redistribute to lists, requires prior specific permission and/or a fee. Request permissions from permissions@acm.org.

STOC '21, June 21-25, 2021, Virtual, Italy

(C) 2021 Association for Computing Machinery.

ACM ISBN 978-1-4503-8053-9/21/06 ..\$15.00

https://doi.org/10.1145/3406325.3451037

\subsection{Previous Work}

To solve the problem, two methods are often used in the literature: the visibility graph and the continuous Dijkstra. The visibility graph method is to first construct the visibility graph of the vertices of $\mathcal{P}$ along with $s$ and $t$, and then run Dijkstra's shortest path algorithm on the graph to find a shortest $s-t$ path. The best algorithms for constructing the visibility graph run in $O(n \log n+K)$ time [18] or in $O(n+h \log h+K)$ time [7,28] for any constant $\epsilon>0$ after the free space $\mathcal{F}$ is triangulated, where $K$ is the number of edges of the visibility graph. Because $K=\Omega\left(n^{2}\right)$ in the worst case, the visibility graph method inherently takes quadratic time. To deal with the case where $h$ is relatively small comparing to $n$, a variation of the visibility graph method was proposed that is to first construct a so-called tangent graph and then find a shortest $s$ - $t$ path in the graph. Using this method, a shortest $s-t$ path can be found in $O(n+$ $h \log h+K^{\prime}$ ) time [6] after $\mathcal{F}$ is triangulated, where $K^{\prime}$ may be considered as the number of tangents among obstacles of $\mathcal{P}$ and $K^{\prime}=O\left(h^{2}\right)$. Note that triangulating $\mathcal{F}$ can be done in $O(n \log n)$ time or in $O\left(n+h \log ^{1+\epsilon} h\right)$ time for any small $\epsilon>0$ [2]. Hence, the runtime of the algorithm in [6] is still quadratic in the worst case.

Using the continuous Dijkstra method, Mitchell [36] made a breakthrough and achieved the first subquadratic algorithm of $O\left(n^{3 / 2+\epsilon}\right)$ time for any constant $\epsilon>0$. Also using the continuous Dijkstra approach plus a novel conforming subdivision of the free space, Hershberger and Suri [23] presented an algorithm of $O(n \log n)$ time and $O(n \log n)$ space; the runtime is optimal when $h=\Theta(n)$ as $\Omega(n+h \log h)$ is a lower bound (which can be obtained by a reduction from sorting; e.g., see Theorem 3 [14] for a similar reduction). By modifying Hershberger and Suri's algorithm, Wang [44] recently reduced the space to $O(n)$ while the runtime is still $O(n \log n)$. In addition, Inkulu, Kapoor, and Maheshwari [26] announced an algorithm of $O(n+h \log h \log n)$ time and $O(n)$ space, which is also based on the continuous Dijkstra approach.

All three continuous Dijkstra algorithms [23, 36, 44] construct the shortest path map, denoted by $\operatorname{SPM}(s)$, for a source point $s$. $\operatorname{SPM}(s)$ is of $O(n)$ size and can be used to answer shortest path queries. By building a point location data structure on $\operatorname{SPM}(s)$ in $O(n)$ time $[16,30]$, given a query point $t$, the shortest path length from $s$ to $t$ can be computed in $O(\log n)$ time and a shortest $s-t$ path can be output in time linear in the number of edges of the path.

The problem setting for $\mathcal{P}$ is usually referred to as polygonal domains or polygons with holes in the literature. The problem in simple polygons is relatively easier [19-22, 32]. Guibas et al. [20] presented an algorithm that can construct a shortest path map in linear time. For two-point shortest path query problem where both $s$ and $t$ are query points, Guibas and Hershberger [19,21] built a data structure in linear time such that each query can be answered 
in $O(\log n)$ time. In contrast, the two-point query problem in polygonal domains is much more challenging: to achieve $O(\log n)$ time queries, the current best result uses $O\left(n^{11}\right)$ space [10]; alternatively Chiang and Mitchell [10] gave a data structure of $O\left(n+h^{5}\right)$ space with $O(h \log n)$ query time.

The $L_{1}$ shortest path problem where the path length is measured in the $L_{1}$ metric also attracted much attention, e.g., [1, 9, 12, 13, 33, 35]. For polygons with holes, Mitchell [33, 35] gave an algorithm that can build a shortest path map for a source point in $O(n \log n)$ time and $O(n)$ space; for small $h$, Chen and Wang [9] proposed an algorithm of $O(n+h \log h)$ time and $O(n)$ space, after the free space is triangulated. For simple polygons, Bae and Wang [1] built a data structure in $O(n)$ time that can answer each two-point shortest path query in $O(\log n)$ time. The two-point query problem in polygons with holes was also studied $[4,5,43]$. To achieve $O(\log n)$ query time, the best result uses $O\left(n+h^{2} \log ^{3} h / \log \log h\right)$ space [43].

\subsection{Our Result}

In this paper, we show that the problem of finding an Euclidean shortest path among obstacles in $\mathcal{P}$ is solvable in $O(n+h \log h)$ time and $O(n)$ space, after a triangulation of the free space $\mathcal{F}$ is given. If the time for triangulating $\mathcal{F}$ is included and the triangulation algorithm in [2] is used, then the total time of the algorithm is $O\left(n+h \log ^{1+\epsilon} h\right)$, for any constant $\epsilon>0 .{ }^{1}$ With the assumption that the triangulation could be done in $O(n+h \log h)$ time, which has been an open problem and is beyond the scope of this paper, our result settles Problem 21 in The Open Problem Project [15] Our algorithm actually constructs the shortest path map $\operatorname{SPM}(s)$ for the source point $s$ in $O(n+h \log h)$ time and $O(n)$ space. We give an overview of our approach below. The high-level scheme of our algorithm is similar to that for the $L_{1}$ case [43] in the sense that we first solve the convex case where all obstacles of $\mathcal{P}$ are convex and then extend the algorithm to the general case using the extended corridor structure [4, 7-9, 27-29, 37].

The convex case. We first discuss the convex case. Let $\mathcal{V}$ denote the set of topmost, bottommost, leftmost, and rightmost vertices of all obstacles. Hence, $|\mathcal{V}| \leq 4 h$. Using the algorithm of Hershberger and Suri [23], we build a conforming subdivision $\mathcal{S}$ on the points of $\mathcal{V}$, without considering the obstacle edges. Since $|\mathcal{V}|=O(h)$, the size of $\mathcal{S}$ is $O(h)$. Then, we insert the obstacle edges into $\mathcal{S}$ to build a conforming subdivision $\mathcal{S}^{\prime}$ of the free space. The subdivision $\mathcal{S}^{\prime}$ has $O(h)$ cells (in contrast, the conforming subdivision of the free space in [23] has $O(n)$ cells). Unlike the subdivision in [23] where each cell is of constant size, here the size of each cell of $\mathcal{S}^{\prime}$ may not be constant but its boundary consists of $O(1)$ transparent edges and $O(1)$ convex chains (each of which belongs to the boundary of an obstacle of $\mathcal{P}$ ). Like the subdivision in [23], each transparent edge $e$ of $\mathcal{S}^{\prime}$ has a well-covering region $\mathcal{U}(e)$. In particular, for each transparent edge $f$ on the boundary of $\mathcal{U}(e)$, the shortest path distance between $e$ and $f$ is at least $2 \cdot \max \{|e|,|f|\}$. Using $\mathcal{S}^{\prime}$ as a guidance, we run the continuous Dijkstra algorithm as in [23] to expand the wavefront, starting from the source point $s$. A main challenge our algorithm needs to overcome (which is also

\footnotetext{
${ }^{1}$ If randomization is allowed, the algorithm of Clarkson, Cole, and Tarjan [11] can compute a triangulation in $O\left(n \log ^{*} n+h \log h\right)$ expected time. If all obstacles of $\mathcal{P}$ are convex, then the triangulation can be done in $O(n+h \log h)$ time [25].
}

a main difference between our algorithm and that in [23]) is that each cell in our subdivision $\mathcal{S}^{\prime}$ may not be of constant size. One critical property our algorithm relies on is that the boundary of each cell of $\mathcal{S}^{\prime}$ has $O(1)$ convex chains. Our strategy is to somehow treat each such convex chain as a whole. We also borrow some idea from the algorithm of Hershberger, Suri, and Yildız [24] for computing shortest paths among curved obstacles. To guarantee the $O(n+h \log h)$ time, some global charging analysis is used. In addition, the tentative prune-and-search technique of Kirkpatrick and Snoeyink [31] is applied to perform certain operations related to bisectors, in logarithmic time each. Finally, the techniques of Wang [44] are utilized to reduce the space to $O(n)$. All these efforts lead to an $O(n+h \log h)$ time and $O(n)$ space algorithm to construct the shortest path map $\operatorname{SPM}(s)$ for the convex case.

The general case. We extend the convex case algorithm to the general case where obstacles may not be convex. To this end, we resort to the extended corridor structure of $\mathcal{P}$, which was used before for reducing the time complexities from $n$ to $h$, e.g., [4, 79, 27-29, 37]. The structure partitions the free space $\mathcal{F}$ into an ocean $\mathcal{M}, O(n)$ bays, and $O(h)$ canals. The boundary of $\mathcal{M}$ consists of $O(h)$ convex chains. A critical property related to shortest paths is that if both $s$ and $t$ are in $\mathcal{M}$, then any shortest $s-t$ path must be in the union of $\mathcal{M}$ and all corridor paths, each of which is contained in a canal. As the boundary of $\mathcal{M}$ consists of $O(h)$ convex chains, by incorporating all corridor paths, we can easily extend our convex case algorithm to computing $\operatorname{SPM}(\mathcal{M})$, the shortest path map $\operatorname{SPM}(s)$ restricted to $\mathcal{M}$, i.e., $\operatorname{SPM}(\mathcal{M})=\operatorname{SPM}(s) \cap \mathcal{M}$. To compute the entire map $\operatorname{SPM}(s)$, we expand $\operatorname{SPM}(\mathcal{M})$ to all bays and canals through their gates (i.e., their common edges shared with $\mathcal{M}$; a bay has one gate while a canal has two gates). For this, we process each bay/canal individually. For each bay/canal $C$, expanding the map into $C$ is actually a special case of the (additively)-weighted geodesic Voronoi diagram problem on a simple polygon where all sites are outside $C$ and can influence $C$ only through its gates. In summary, after a triangulation of $\mathcal{F}$ is given, building $\operatorname{SPM}(\mathcal{M})$ takes $O(n+h \log h)$ time, and expanding $\operatorname{SPM}(\mathcal{M})$ to all bays and canals takes additional $O(n+h \log h)$ time. The total space is $O(n)$.

It should be noted that a fundamental difference between our algorithm and the $O(n+h \log h \log n)$-time algorithm of [26] is that our algorithm follows the framework of Hershberger and Suri [23] (as well as [24]) by using a conforming subdivision of the free space as an underlying structure to guide the wavefront expansion while the algorithm of [26] does not use the conforming subdivision (it instead uses the triangulation as well as the corridor structure of the free space). In addition, our algorithm computes a shortest path map for the source point $s$ while the algorithm of [26] computes a shortest path from $s$ to a single point $t$.

The rest of the paper is organized as follows. Section 2 introduces some notation and concepts. Section 3 presents the algorithm for the convex case. The general case is discussed in Section 4. Due to the space limit, we have to omit many proofs and details, all of which can be found in the full paper.

\section{PRELIMINARIES}

For two points $a$ and $b$ in the plane, denote by $\overline{a b}$ the line segment with $a$ and $b$ as endpoints; denote by $|\overline{a b}|$ the length of the segment. 
For any two points $s$ and $t$ in the free space $\mathcal{F}$, we use $\pi(s, t)$ to denote a shortest path from $s$ to $t$ in $\mathcal{F}$. In the case where shortest paths are not unique, $\pi(s, t)$ may refer to an arbitrary one. Denote by $d(s, t)$ the length of $\pi(s, t)$; we call $d(s, t)$ the geodesic distance between $s$ and $t$. For two line segments $e$ and $f$ in $\mathcal{F}$, their geodesic distance is defined to be the minimum geodesic distance between any point on $e$ and any point on $f$, i.e., $\min _{s \in e, t \in f} d(s, t)$; by slightly abusing the notation, we use $d(e, f)$ to denote their geodesic distance. For any path $\pi$ in the plane, we use $|\pi|$ to denote its length.

For any region $A$ in the plane, let $\partial A$ denote its boundary. We use $\partial \mathcal{P}$ to denote the union of the boundaries of all obstacles of $\mathcal{P}$.

Throughout the paper, we use $s$ to refer to the source point. For convenience, we consider $s$ as a degenerate obstacle in $\mathcal{P}$. We often refer to the vertices of $\mathcal{P}$ as obstacle vertices and refer to the edges of $\mathcal{P}$ as obstacle edges. For any point $t \in \mathcal{F}$, we call the adjacent vertex of $t$ in $\pi(s, t)$ the anchor of $t$ in $\pi(s, t) .^{2}$

The shortest path map $\operatorname{SPM}(s)$ of $s$ is a decomposition of the free space $\mathcal{F}$ into maximal regions such that all points in each region $R$ have the same anchor $[23,34]$ in their shortest paths from $s$; e.g., see Fig. 1. Each edge of $\operatorname{SPM}(s)$ is either an obstacle edge fragment or a bisecting-curve $e^{3}$, which is the locus of points $p$ with $d(s, u)+|\overline{p u}|=d(s, v)+|\overline{p v}|$ for two obstacle vertices $u$ and $v$. Each bisecting-curve is in general a hyperbola; a special case happens if one of $u$ and $v$ is the anchor of the other, in which case their bisecting-curve is a straight line. Following the notation in [17], we differentiate between two types of bisecting-curves: walls and windows. A bisecting curve is a wall if there exist two topologically different shortest paths from $s$ to each point of the edge; otherwise (i.e., the above special case) it is a window (e.g., see Fig. 1).

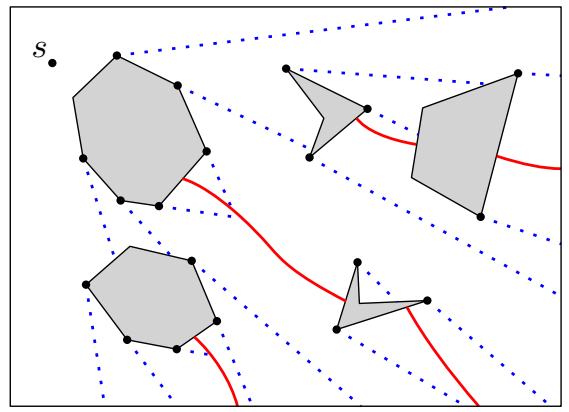

Figure 1: Illustrating the shortest path map $S P M(s)$. The solid (red) curves are walls and the (blue) dotted segments are windows. The anchor of each cell is shown with a black point.

We make a general position assumption that for each obstacle vertex $v$, there is a unique shortest path from $s$ to $v$, and for any point $p$ in the plane, there are at most three different shortest paths from $s$ to $p$. The assumption assures that each vertex of $\operatorname{SPM}(s)$ has degree at most three, and there are at most three bisectors of SPM $(s)$ intersecting at a common point, which is sometimes called a triple point in the literature [17]. A curve in the plane is $x$-monotone if its intersection with any vertical line is connected; the $y$-monotone

\footnotetext{
${ }^{2}$ Usually "predecessor" is used in the literature instead of "anchor", but here we reserve "predecessor" for other purpose.

${ }^{3}$ This is usually called bisector in the literature. Here we reserve the term "bisector" to be used later.
}

is defined similarly. A curve is $x y$-monotone if it is both $x$ - and $y$-monotone. Observe that $n+h \log n=O(n+h \log h)$, which will be used throughout the paper without explicitly mentioning it again.

\section{THE CONVEX CASE}

In this section, we present our algorithm for the convex case where all obstacles of $\mathcal{P}$ are convex.

For each obstacle $P \in \mathcal{P}$, the topmost, bottommost, leftmost, and rightmost vertices of $P$ are called rectilinear extreme vertices. The four rectilinear extreme vertices partition $\partial P$ into four portions and each portion is called an elementary chain, which is convex and $x y$ monotone. For technical reason that will be clear later, we assume that each rectilinear extreme vertex $v$ belongs to the elementary chain counterclockwise of $v$ with respect to the obstacle (i.e., $v$ is the clockwise endpoint of the chain; e.g., see Fig. 2). We use elementary chain fragment to refer to a portion of an elementary chain.

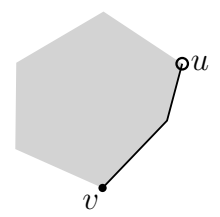

Figure 2: Illustrating an elementary chain (the thick segments), which contains the vertex $v$ but not $u$.

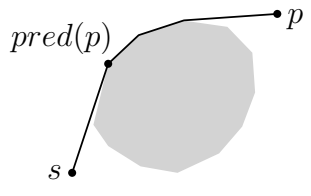

(a)

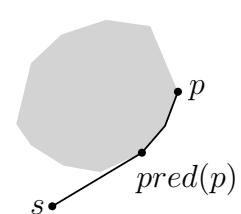

(b)
Figure 3: Illustrating the predecessor.

We introduce some notation below that is similar in spirit to those from [24] for shortest paths among curved obstacles.

Consider a shortest path $\pi(s, p)$ from $s$ to a point $p$ in the free space $\mathcal{F}$. It is not difficult to see that $\pi(s, p)$ is a sequence of elementary chain fragments and common tangents between obstacles of $\mathcal{P} \cup\{p\}$. We define the predecessor of $p$, denoted by pred $(p)$, to be the initial vertex of the last elementary chain fragment in $\pi(s, p)$ (e.g., see Fig. $3(\mathrm{a}))$. Note that since each rectilinear extreme vertex belongs to a single elementary chain, $\operatorname{pred}(p)$ in $\pi(s, p)$ is unique. A special case happens if $p$ is a rectilinear extreme vertex and $\pi(s, p)$ contains a portion of an elementary chain $A$ clockwise of $p$. In this case, we let pred( $p)$ be endpoint of the fragment of $A$ in $\pi(s, p)$ other than $p$ (e.g., see Fig. $3(\mathrm{~b})$ ); in this way, $\operatorname{pred}(p)$ is unique in $\pi(s, p)$. Note that $p$ may still have multiple predecessors if there are multiple shortest paths from $s$ to $p$. Intuitively, the reason we define predecessors as above is to treat each elementary chain somehow as a whole, which is essential for reducing the runtime of the algorithm from $n$ to $h$. 
The rest of this section is organized as follows. In Section 3.1, we compute a conforming subdivision $\mathcal{S}^{\prime}$ of the free space $\mathcal{F}$. Section 3.2 introduces some basic concepts and notation for our algorithm. The wavefront expansion algorithm is presented in Section 3.3, with two key subroutines of the algorithm described in Section 3.4 and Section 3.5, respectively. Section 3.6 analyzes the time complexity of the algorithm. Using the information computed by the wavefront expansion algorithm, Section 3.7 constructs the shortest path map SPM $(s)$. The overall algorithm runs in $O(n+h \log h)$ time and space. Section 3.8 reduces the space to $O(n)$ while keeping the same runtime, by using the techniques from Wang [44].

\subsection{Computing a Conforming Subdivision}

Let $\mathcal{V}$ denote the set of the rectilinear extreme vertices of all obstacles of $\mathcal{P}$. Hence, $|\mathcal{V}|=O(h)$. Using the algorithm of algorithm of Hershberger and Suri [23] (called the HS algorithm), we build a conforming subdivision $\mathcal{S}$ with respect to the vertices of $\mathcal{V}$, without considering the obstacle edges.

The subdivision $\mathcal{S}$, which is of size $O(h)$, is a quad-tree-style subdivision of the plane into $O(h)$ cells. Each cell of $\mathcal{S}$ is a square or a square annulus (i.e., an outer square with an inner square hole). Each vertex of $\mathcal{V}$ is contained in the interior of a square cell and each square cell contains at most one vertex of $\mathcal{V}$. Each edge $e$ of $\mathcal{S}$ is axis-parallel and well-covered, i.e., there exists a set $C(e)$ of $O(1)$ cells of $\mathcal{S}$ such that their union $\mathcal{U}(e)$ contains $e$ with the following properties: (1) the total complexity of all cells of $C(e)$ is $O(1)$ and thus the size of $\mathcal{U}(e)$ is $O(1)$; (2) for any edge $f$ of $\mathcal{S}$ that is on $\partial \mathcal{U}(e)$ or outside $\mathcal{U}(e)$, the Euclidean distance between $e$ and $f$ (i.e., the minimum $|\overline{p q}|$ among all points $p \in e$ and $q \in f$ ) is at least $2 \cdot \max \{|e|,|f|\} ;(3) \mathcal{U}(e)$, which is called the well-covering region of $e$, contains at most one vertex of $\mathcal{V}$. In addition, each cell $c$ of $\mathcal{S}$ has $O(1)$ edges on its boundary with the following uniform edge property: the lengths of the edges on the boundary of $c$ differ by at most a factor of 4, regardless of whether $c$ is a square or square annulus. $\mathcal{S}$ can be computed in $O(h \log h)$ time and $O(h)$ space [23].

Next we insert the obstacle edges into $\mathcal{S}$ to produce a conforming subdivision $\mathcal{S}^{\prime}$ of the free space $\mathcal{F} . \mathcal{S}^{\prime}$ has two types of edges: those introduced by the subdivision construction (which are in $\mathcal{F}$ ) and the obstacle edges; we call the former the transparent edges (which are axis-parallel) and the latter the opaque edges. The definition of $\mathcal{S}^{\prime}$ is similar to the conforming subdivision used in the HS algorithm. A main difference is that here endpoints of each obstacle edge may not be in $\mathcal{V}$, a consequence of which is that each cell of $\mathcal{S}^{\prime}$ may not be of constant size (while each cell in the subdivision of the HS algorithm is of constant size). However, each cell $c$ of $\mathcal{S}^{\prime}$ has the following property that is critical to our algorithm: The boundary $\partial c$ consists of $O(1)$ transparent edges and $O(1)$ convex chains (each of which is a portion of an elementary chain).

More specifically, $\mathcal{S}^{\prime}$ is a subdivision of $\mathcal{F}$ into $O(h)$ cells. Each cell of $\mathcal{S}^{\prime}$ is one of the connected components formed by intersecting $\mathcal{F}$ with an axis-parallel rectangle (which is the union of a set of adjacent cells of $\mathcal{S}$ ) or a square annulus of $\mathcal{S}$. Each cell of $\mathcal{S}^{\prime}$ contains at most one vertex of $\mathcal{V}$. Each vertex of $\mathcal{V}$ is incident to a transparent edge. Each transparent edge $e$ of $\mathcal{S}^{\prime}$ is well-covered, i.e., there is a set $\mathcal{C}(e)$ of $O(1)$ cells whose union $\mathcal{U}(e)$ contains $e$ with the following property: for each transparent edge $f$ on $\partial \mathcal{U}(e)$, the geodesic distance $d(e, f)$ between $e$ and $f$ is at least $2 \cdot \max \{|e|,|f|\}$. $\mathcal{U}(e)$ is called the well-covering region of $e$ and contains at most one vertex of $\mathcal{V}$. Note that $\mathcal{S}^{\prime}$ has $O(h)$ transparent edges.

Below we show how $\mathcal{S}^{\prime}$ is produced from $\mathcal{S}$. The procedure is similar to that in the HS algorithm. We overlay the obstacle edges on top of $\mathcal{S}$ to obtain a subdivision $\mathcal{S}_{\text {overlay }}$. Because each edge of $\mathcal{S}$ is axis-parallel and all obstacle edges constitute a total of $O(h)$ elementary chains, each of which is $x y$-monotone, $\mathcal{S}_{\text {overlay }}$ has $O\left(h^{2}\right)$ faces. We say that a face of $\mathcal{S}_{\text {overlay }}$ is interesting if its boundary contains a vertex of $\mathcal{V}$ or a vertex of $\mathcal{S}$. We keep intact the interesting faces of $\mathcal{S}_{\text {overlay }}$ while deleting every edge fragment of $\mathcal{S}$ not on the boundary of any interesting cell. Further, for each cell $c$ containing a vertex $v \in \mathcal{V}$, we partition $c$ by extending vertical edges from $v$ until the boundary of $c$. This divides $c$ into at most three subcells. Finally we divide each of the two added edges incident to $v$ into segments of length at most $\delta$, where $\delta$ is the length of the shortest edge on the boundary of $c$. By the uniform edge property of $\mathcal{S}, \partial c$ has $O(1)$ edges, whose lengths differ by at most a factor of 4 ; hence dividing the edges incident to $v$ as above produces only $O(1)$ vertical edges. The resulting subdivision is $\mathcal{S}^{\prime}$.

As mentioned above, the essential difference between our subdivision $\mathcal{S}^{\prime}$ and the one in the HS algorithm is that the role of an obstacle edge in the HS algorithm is replaced by an elementary chain. Therefore, each opaque edge in the subdivision of the HS algorithm becomes an elementary chain fragment in our case. Hence, by the same analysis as in the HS algorithm (see Lemma 2.2 [23]), $\mathcal{S}^{\prime}$ has the properties as described above and the well-covering region $\mathcal{U}(e)$ of each transparent edge $e$ of $\mathcal{S}^{\prime}$ is defined in the same way as in the HS algorithm.

LEMmA 3.1. The conforming subdivision $\mathcal{S}^{\prime}$ can be constructed in $O(n+h \log h)$ time and $O(n)$ space.

Note that although $\mathcal{S}^{\prime}$ is defined with the help of $\mathcal{S}_{\text {overlay }}, \mathcal{S}^{\prime}$ is constructed directly without computing $\mathcal{S}_{\text {overlay }}$ first.

\subsection{Basic Concepts and Notation}

Our shortest path algorithm uses the continuous Dijkstra method. We initially generate a wavefront from $s$, which is a circle centered at $s$. During the algorithm, the wavefront consists of all points of $\mathcal{F}$ with the same geodesic distance from $s$. We expand the wavefront until all points of the free space are covered. The conforming subdivision $\mathcal{S}^{\prime}$ is utilized to guide the wavefront expansion. Our wavefront expansion algorithm follows the high-level scheme as the HS algorithm. The difference is that our algorithm somehow considers each elementary chain as a whole, which is in some sense similar to the algorithm of Hershberger, Suri, and Yıldız [24] (called the HSY algorithm). Indeed, the HSY algorithm considers each $x y$ monotone convex arc as a whole, but the difference is that each arc in the HSY algorithm is of constant size while in our case each elementary chain may not be of constant size. As such, techniques from both the HS and the HSY algorithms are borrowed.

We use $\tau$ to denote the geodesic distance from $s$ to all points in the wavefront. One may also think of $\tau$ as a parameter representing time. The algorithm simulates the expansion of the wavefront as time increases from 0 to $\infty$. The wavefront comprises a sequence of wavelets, each emanating from a generator. In the HS algorithm, a generator is simply an obstacle vertex. Here, since we want to 
treat an elementary chain as a whole, similar to the HSY algorithm (also similar to the concept "bunch" introduced in [26]), we define a generator as a couple $\alpha=(A, a)$, where $A$ is an elementary chain and $a$ is an obstacle vertex on $A$, and further a clockwise or counterclockwise direction of $A$ is designated for $\alpha$; $a$ has a weight $w(a)$ (one may consider $w(a)$ as the geodesic distance between $s$ and $a$ ). We call $a$ the initial vertex of the generator $\alpha$.

We say a point $q$ is reachable by a generator $\alpha=(A, a)$ if one can draw a path in $\mathcal{F}$ from $a$ to $q$ by following $A$ in the designated direction to a vertex $v$ on $A$ such that $\overline{v q}$ is tangent to $A$ and then following the segment $\overline{v q}$ (e.g., see Fig. 4). The (weighted) distance between the generator $\alpha$ and $q$ is the length of this path plus $w(a)$; by slightly abusing the notation, we use $d(\alpha, q)$ to denote the distance. From the definition of reachable points, the vertex $a$ partitions $A$ into two portions and only the portion following the designated direction is relevant (in Fig. 4, only the portion containing the vertex $v$ is relevant). Henceforth, unless otherwise stated, we use $A$ to refer to its relevant portion only and call $A$ the underlying chain of $\alpha$. In this way the initial vertex $a$ becomes an endpoint of $A$. For convenience, sometimes we do not differentiate $\alpha$ and $A$. For example, when we say "the tangent from $q$ to $\alpha$ ", we mean "the tangent from $q$ to $A$ "; "a vertex of $\alpha$ " refers to "a vertex of $A$ ".

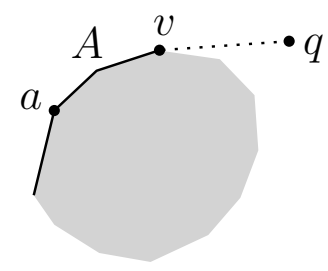

Figure 4: Illustrating a generator $\alpha=(A, a)$. $A$ consists of the thick segments, and is designated clockwise direction around the obstacle. $q$ is a reachable point through $v$.

The wavefront can thus be represented by the sequence of generators of its wavelets. A wavelet generated by a generator $\alpha=(A, a)$ at time $\tau$ is a contiguous set of reachable points $q$ such that $d(\alpha, q)=\tau$ and $d\left(\alpha^{\prime}, q\right) \geq \tau$ for all other generators $\alpha^{\prime}$ in the wavefront; we also say that $q$ is claimed by $\alpha$. Note that as $A$ may not be of constant size, a wavelet may not be of constant size either; it actually consists of a contiguous sequence of circular arcs centered at the obstacle vertices $A$ (e.g., see Fig. 5). If a point $q$ is claimed by $\alpha$, then $d(s, q)=d(\alpha, q)=\tau$ and the predecessor $\operatorname{pred}(q)$ of $q$ is $a$; sometimes for convenience we also say that the generator $\alpha$ is the predecessor of $q$. If $q$ is on an elementary chain $A^{\prime}$ and the tangent from $q$ to $a$ is also tangent to $A^{\prime}$, then a new generator $\left(A^{\prime}, q\right)$ is added to the wavefront (e.g., see Fig. 6(a)). A special case happens when $q$ is the counterclockwise endpoint of $A$ (and thus $q$ does not belong to $A$ ); in this case, a new generator $\alpha^{\prime}=\left(A^{\prime}, q\right)$ is also added, where $A^{\prime}$ is the elementary chain that contains $q$ (e.g., see Fig. 6(b))

As $\tau$ increases, the points bounding the adjacent wavelets trace out the bisectors that form the edges of the shortest path map $\operatorname{SPM}(s)$. The bisector between the wavelets of two generators $\alpha$ and $\alpha^{\prime}$, denoted by $B\left(\alpha, \alpha^{\prime}\right)$, consists of points $q$ with $d(\alpha, q)=d\left(\alpha^{\prime}, q\right)$. Note that since $\alpha$ and $\alpha^{\prime}$ may not be of constant size, $B\left(\alpha, \alpha^{\prime}\right)$ may not be of constant size either (in contrast, the bisector in HSY's algorithm is always of constant size; note that a similar concept

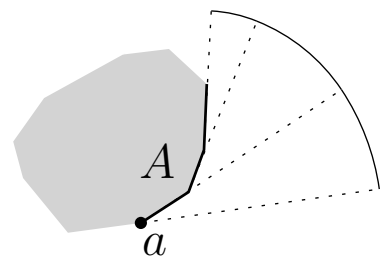

Figure 5: A wavelet generated by $\alpha=(A, q)$, designated counterclockwise direction. The wavelet has three pieces each of which is a circular arc.

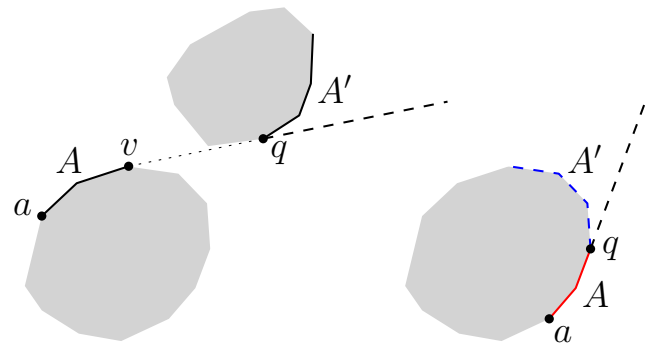

(a)

(b)

Figure 6: Illustrating a new generator $\alpha^{\prime}=\left(A^{\prime}, q\right)$. (a) A general case where both $A$ and $A^{\prime}$ are marked with thick segments. (b) A special case where $A$ is marked with solid (red) segments and $A^{\prime}$ is marked with dashed (blue) segments.

"I-curves" is introduced in [26]). More specifically, $B\left(\alpha, \alpha^{\prime}\right)$ has multiple pieces each of which is a hyperbola defined by two obstacle vertices $v \in \alpha$ and $v^{\prime} \in \alpha^{\prime}$ such that the hyperbola consists of all points that have two shortest paths from $s$ with $v$ and $v^{\prime}$ as the anchors, respectively (e.g., see Fig. 7). A special case happens if $\alpha^{\prime}$ is a generator created by the wavelet of $\alpha$, such as in Fig. 6(a), then $B\left(\alpha, \alpha^{\prime}\right)$ is the half-line extended from $q$ along the direction from $v$ to $q$ (the dashed segment in Fig. 6(a)); we call such a bisector an $e x-$ tension bisector. Note that in the case illustrated in Fig. 6(b), $B\left(\alpha, \alpha^{\prime}\right)$, which is also an extension bisector, is the half-line extended from $q$ along the direction from $v$ to $q$ (the dashed segment in Fig. 6(b)), where $v$ is the obstacle vertex adjacent to $q$ in $A$.

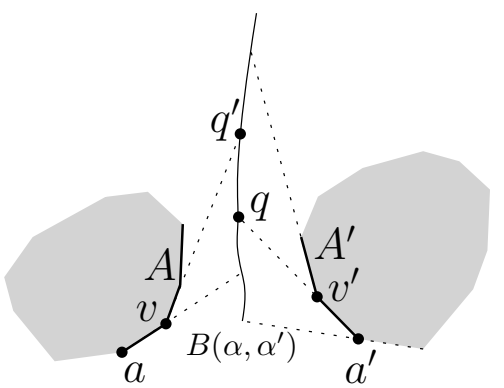

Figure 7: A bisector $B\left(\gamma, \gamma^{\prime}\right)$ defined by two generators $\gamma=$ $(A, q)$ and $\gamma=\left(A^{\prime}, q^{\prime}\right)$. The portion between $q$ and $q^{\prime}$ is a hyperbola defined by $v$ and $v^{\prime}$.

A wavelet gets eliminated from the wavefront if the two bisectors bounding it intersect, which is called a bisector event. Specifically, if 
$\alpha_{1}, \alpha$, and $\alpha_{2}$ are three consecutive generators of the wavefront, the wavelet generated by $\alpha$ will be eliminated when $B\left(\alpha_{1}, \alpha\right)$ intersects $B\left(\alpha, \alpha_{2}\right)$; e.g., see Fig. 8 . Wavelets are also eliminated by collisions with obstacles and other wavelets in front of it. If a bisector $B\left(\alpha, \alpha^{\prime}\right)$ intersects an obstacle, their intersection is also a bisector event.

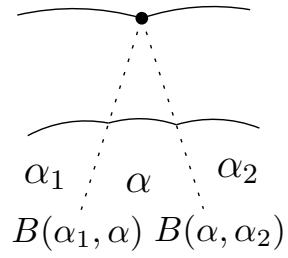

Figure 8: Illustrating the intersection of two bisectors.

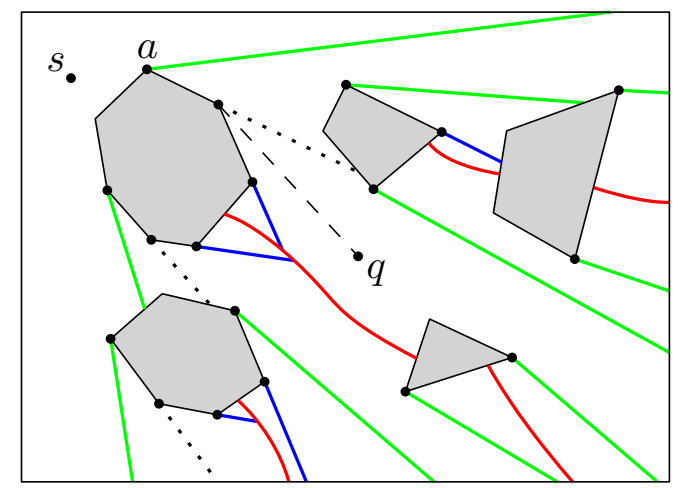

Figure 9: Illustrating the map $\operatorname{SPM}(s)$. The three dotted black segments are windows that are not in $S P M^{\prime}(s)$; removing them becomes $S P M^{\prime}(s)$. The red curves are non-extension bisectors. The green and blue segments are extension bisectors, where the green and blue ones are Type (a) and (b) as illustrated in Fig. 6 . The predecessor in each cell is also shown with a black point. For example, all points in the cell containing $q$ have $a$ as their predecessor.

Let $S P M^{\prime}(s)$ be the subdivision of $\mathcal{F}$ by the bisectors of all generators (e.g. see Fig. 9). The intersections of bisectors and intersections between bisectors and obstacle edges are vertices of $S P M^{\prime}(s)$. Each bisector connecting two vertices is an edge of $\operatorname{SPM}^{\prime}(s)$, called a bisector edge. As discussed before, a bisector, which consists of multiple hyperbola pieces, may not be of constant size. Hence, a bisector edge $e$ of $S P M^{\prime}(s)$ may not be of constant size. For differentiation, we call each hyperbola piece of $e$, a hyperbolic-arc. In addition, if the boundary of an obstacle $P$ contains more than one vertex of $S P M^{\prime}(s)$, then the chain of edges of $\partial P$ connecting two adjacent vertices of $S P M^{\prime}(s)$ also forms an edge of $S P M^{\prime}(s)$, called a convex-chain edge. $\operatorname{SPM}^{\prime}(s)$ is very similar to $\operatorname{SPM}(s)$ : Each extension bisector edge of $\operatorname{SPM}^{\prime}(s)$ is a window of $\operatorname{SPM}(s)$; the union of all non-extension bisector edges of $S P M^{\prime}(s)$ is exactly the union of all walls of $\operatorname{SPM}(s) \cdot S P M^{\prime}(s)$ can be obtained from $\operatorname{SPM}(s)$ by removing all windows except those that are extension bisectors of $S P M^{\prime}(s)$.

We can show the following: the combinatorial size of $S P M^{\prime}(s)$ is $O(n)$ and $S P M^{\prime}(s)$ has $O(h)$ faces, vertices, and edges. In particular, $S P M^{\prime}(s)$ has $O(h)$ bisector intersections and $O(h)$ intersections between bisectors and obstacle edges. Therefore, there are $O(h)$ bisector events and $O(h)$ generators in $S P M^{\prime}(s)$.

By the definition of $S P M^{\prime}(s)$, each cell $C$ of $S P M^{\prime}(s)$ has a unique generator $\alpha=(A, a)$, all points of the cell are reachable from the generator, and $a$ is the predecessor of all points of $C$ (e.g., see Fig. 9; all points in the cell containing $q$ have $a$ as their predecessor). Hence, for any point $q \in C$, we can compute $d(s, q)=d(\alpha, q)$ by computing the tangent from $q$ to $A$. Thus, $S_{P M}^{\prime}(s)$ can also be used to answer shortest path queries. In face, given $S P M^{\prime}(s)$, we can construct $\operatorname{SPM}(s)$ in additional $O(n)$ time by inserting the windows of $\operatorname{SPM}(s)$ to $S P M^{\prime}(s)$. Hence, it suffices to compute $\operatorname{SPM}^{\prime}(s)$ below.

\subsection{The Wavefront Expansion Algorithm}

To simulate the wavefront expansion, we compute the wavefront passing through each transparent edge of the conforming subdivision $\mathcal{S}^{\prime}$. As in the HS algorithm, since it is difficult to compute a true wavefront for each transparent edge $e$ of $\mathcal{S}^{\prime}$, a key idea is to compute two one-sided wavefronts (called approximate wavefronts) for $e$, each representing the wavefront coming from one side of $e$. Intuitively, an approximate wavefront from one side of $e$ is what the true wavefront would be if the wavefront were blocked off at $e$ by considering $e$ as an opaque segment (with open endpoints).

In the following, unless otherwise stated, a wavefront at a transparent edge $e$ of $\mathcal{S}^{\prime}$ refers to an approximate wavefront. Let $W(e)$ denote a wavefront at $e$. To make the description concise, as there are two wavefronts at $e$, depending on the context, $W(e)$ may refer to both wavefronts, i.e., the discussion on $W(e)$ applies to both wavefronts. For example, "compute the wavefronts $W(e)$ " means "compute both wavefronts at $e$ ".

For each transparent edge $e$ of $\mathcal{S}^{\prime}$, define input(e) as the set of transparent edges on the boundary of the well-covering region $\mathcal{U}(e)$, and define output $(e)=\{g \mid e \in \operatorname{input}(g)\} \cup \operatorname{input}(e)$. Because $\partial \mathcal{U}\left(e^{\prime}\right)$ for each transparent edge $e^{\prime}$ of $\mathcal{S}^{\prime}$ has $O(1)$ transparent edges, both $\mid$ input $(e) \mid$ and $\mid$ output $(e) \mid$ are $O(1)$.

The wavefront propagation and merging procedures. The wavefronts $W(e)$ at $e$ are computed from the wavefronts at edges of input (e); this guarantees the correctness because $e$ is in $\mathcal{U}(e)$ (and thus any shortest path $\pi(s, p)$ must cross some edge $f \in \operatorname{input}(e)$ for any point $p \in e)$. After the wavefronts $W(e)$ at $e$ are computed, they will pass to the edges of output $(e)$. Also, the geodesic distances from $s$ to both endpoints of $e$ will be computed. Recall that $\mathcal{V}$ is the set of rectilinear extreme vertices of all obstacles and each vertex of $\mathcal{V}$ is incident to a transparent edge of $\mathcal{S}^{\prime}$. As such, after the algorithm is finished, geodesic distances from $s$ to all vertices of $\mathcal{V}$ will be available. The process of passing the wavefronts $W(e)$ at $e$ to all edges $g \in$ output $(e)$ is called the wavefront propagation procedure, which will compute the wavefront $W(e, g)$, where $W(e, g)$ is the portion of $W(e)$ that passes to $g$ through the well-covering region $\mathcal{U}(g)$ of $g$ if $e \in \operatorname{input}(g)$ and through $\mathcal{U}(e)$ otherwise (in this case $g \in \operatorname{input}(e))$; whenever the procedure is invoked on $e$, we say that $e$ is processed. The wavefronts $W(e)$ at $e$ are constructed by merging the wavefronts $W(f, e)$ for edges $f \in \operatorname{input}(e)$; this procedure is called the wavefront merging procedure.

The main algorithm. The transparent edges of $\mathcal{S}^{\prime}$ are processed in a rough time order. The wavefronts $W(e)$ of each transparent 
edge $e$ are constructed at the time $\tilde{d}(s, e)+|e|$, where $\tilde{d}(s, e)$ is the minimum geodesic distance from $s$ to the two endpoints of $e$. Define covertime $(e)=\tilde{d}(s, e)+|e|$. The value covertime $(e)$ will be computed during the algorithm. Initially, for each edge $e$ whose well-covering region $\mathcal{U}(e)$ contains $s, W(e)$ and covertime $(e)$ are computed directly (and set covertime $(e)=\infty$ for all other edges). The algorithm maintains a timer $\tau$ and processes the edges $e$ of $\mathcal{S}^{\prime}$ following the order of covertime(e).

The main loop of the algorithm works as follows. As long as $\mathcal{S}^{\prime}$ has an unprocessed transparent edge, we do the following. First, among all unprocessed transparent edges, choose the one $e$ with minimum covertime $(e)$ and set $\tau=\operatorname{covertime}(e)$. Second, call the wavefront merging procedure to construct the wavefronts $W(e)$ from $W(f, e)$ for all edges $f \in \operatorname{input}(e)$ satisfying covertime $(f)<$ covertime $(e)$; compute $d(s, v)$ from $W(e)$ for each endpoint $v$ of $e$. Third, process $e$, i.e., call the wavefront propagation procedure on $W(e)$ to compute $W(e, g)$ for all edges $g \in$ output $(e)$; in particular, compute the time $\tau_{g}$ when the wavefronts $W(e)$ first encounter an endpoint of $g$ and set covertime $(g)=\min \left\{\operatorname{covertime}(g), \tau_{g}+|g|\right\}$.

The details of the wavefront merging procedure and the wavefront propagation procedure will be described in Section 3.4 and Section 3.5 , respectively.

The algorithm correctness. At the time $\tilde{d}(s, e)+|e|$, all edges $f \in$ input (e) whose wavefronts $W(f)$ contribute a wavelet to $W(e)$ must have already been processed. This is due to the property of the well-covering regions of $\mathcal{S}^{\prime}$ that $d(e, f) \geq 2 \cdot \max \{|e|,|f|\}$ since $f$ is on $\partial \mathcal{U}(e)$.

Artificial wavelets. As in the HS algorithm, when a wavefront propagates across $e$, i.e., when $W(e)$ is computed in the wavefront merging procedure, an artificial wavelet is generated at each endpoint $v$ of $e$, with weight $d(s, v)$. This is to eliminate a wavefront from one side of $e$ if it arrives at $e$ later than the wavefront from the other side of $e$.

Topologically different paths. In the wavefront propagation procedure to pass $W(e)$ to edges $g \in$ output $(e), W(e)$ travels through the cells of the well-covering region $\mathcal{U}$ of either $g$ or $e$. Since $\mathcal{U}$ may not be simply connected (e.g., the square-annulus), there may be multiple topologically different shortest paths between $e$ and $g$ inside $\mathcal{U}$; the number of such paths is $O(1)$ as $\mathcal{U}$ is the union of $O(1)$ cells of $\mathcal{S}^{\prime}$. We propagate $W(e)$ in multiple components of $\mathcal{U}$, each corresponding to a topologically different shortest path and defined by the sequence of transparent edges it crosses. These wavefronts are later combined in the wavefront merging step at $g$.

Claiming a point. During the wavefront merging procedure at $e$, we have a set of wavefronts $W(f, e)$ that reach $e$ from the same side for edges $f \in \operatorname{input}(e)$. We say that a wavefront $W(f, e)$ claims a point $p \in e$ if $W(f, e)$ reaches $p$ before any other wavefront. Further, for each wavefront $W(f, e)$, the set of points on $e$ claimed by it forms an interval. Similarly, a wavelet of a wavefront claims a point $p$ of $e$ if the wavelet reaches $p$ before other wavelets of the wavefront, and the set of points on $e$ claimed by the wavelet also forms an interval. For convenience, if a wavelet claims a point, we also say that the generator of the wavelet claims the point.

Before presenting the details of the wavefront merging and propagation procedures in the next two subsections, we first discuss the data structure (for representing elementary chains, generators, and wavefronts) and a monotonicity property of bisectors.

3.3.1 The Data Structure. We use an array to represent each elementary chain. Then, a generator $\alpha=(A, a)$ can be represented by recording the indices of the two end vertices of its underlying chain $A$. In this way, a generator takes $O(1)$ additional space to record and binary search on $A$ can be supported in $O(\log n)$ time.

For a wavefront $W(e)$ of one side of $e$, it is a list of generators $\alpha_{1}, \alpha_{2}, \ldots$ ordered by the intervals of $e$ claimed by these generators. Note that these generators are in the same side of $e$. Formally, we say that a generator $\alpha$ is in one side of $e$ if the initial vertex of $\alpha$ lies in that side of the supporting line of $e$. We maintain these generators by a balanced binary search tree so that the following operations can be supported in logarithmic time each. Let $W$ be a wavefront with generators $\alpha_{1}, \alpha_{2}, \ldots, \alpha_{k}$.

Insert Insert a generator $\alpha$ to $W$. In our algorithm, $\alpha$ is inserted either in the front of $\alpha_{1}$ or after $\alpha_{k}$.

Delete Delete a generator $\alpha_{i}$ from $W$, for any $1 \leq i \leq k$.

Split Split $W$ into two sublists at some generator $\alpha_{i}$ so that the first $i$ generators form a wavefront and the rest form another wavefront.

Concatenate Concatenate $W$ with another list $W^{\prime}$ of generators so that all generators of $W$ are in the front of those of $W^{\prime}$ in the new list.

We will show later that each wavefront involved in our algorithm has $O(h)$ generators. Therefore, each of the above operation can be performed in $O(\log h)$ time. We make the tree fully persistent by path-copying [40]. In this way, each operation on the tree will cost $O(\log h)$ additional space but the older version of the tree will be kept intact (and operations on the old tree can still be supported).

\subsubsection{The Monotonicity Property of Bisectors.}

Lemma 3.2. Suppose $\alpha_{1}=\left(A_{1}, a_{1}\right)$ and $\alpha_{2}=\left(A_{2}, a_{2}\right)$ are two generators on the same side of an axis-parallel line $\ell$ (i.e., $a_{1}$ and $a_{2}$ are on the same side of $\ell$; it is possible that $\ell$ intersects $A_{1}$ and $A_{2}$ ). Then, the bisector $B\left(\alpha_{1}, \alpha_{2}\right)$ intersects $\ell$ at no more than one point.

Corollary 3.3. Suppose $\alpha_{1}=\left(A_{1}, a_{1}\right)$ and $\alpha_{2}=\left(A_{2}, a_{2}\right)$ are two generators both below a horizontal line $\ell$. Then, the portion of the bisector $B\left(\alpha_{1}, \alpha_{2}\right)$ above $\ell$ is $y$-monotone.

Note that similar monotonicity properties of bisectors were also used in previous work, e.g., [23, 24, 26].

\subsection{The Wavefront Merging Procedure}

In this section, we present the details of the wavefront merging procedure. Given all contributing wavefronts $W(f, e)$ of $f \in \operatorname{input}(e)$ for $W(e)$, the goal of the procedure is to compute $W(e)$. The algorithm follows the high-level scheme of the HS algorithm (i.e., Lemma 4.6 [23]) but the implementation details are quite different.

We only consider the wavefronts $W(f, e)$ and $W(e)$ for one side of $e$ since the algorithm for the other side is analogous. Without loss of generality, we assume that $e$ is horizontal and all wavefronts $W(f, e)$ are coming from below $e$. We describe the algorithm for computing the interval of $e$ claimed by $W(f, e)$ if only one other wavefront $W\left(f^{\prime}, e\right)$ is present. The common intersection of these 
intervals of all such $f^{\prime}$ is the interval of $e$ claimed by $W(f, e)$. Since $|\operatorname{input}(e)|=O(1)$, the number of such $f^{\prime}$ is $O(1)$.

We first determine whether the claim of $W(f, e)$ is to the left or right of that of $W\left(f^{\prime}, e\right)$. To this end, depending on whether both $W(f, e)$ and $W\left(f^{\prime}, e\right)$ reach the left endpoint $v$ of $e$, there are two cases. Note that the intervals of $e$ claimed by $W(f, e)$ and $W\left(f^{\prime}, e\right)$ are available from $W(f, e)$ and $W\left(f^{\prime}, e\right)$; let $I_{f}$ and $I_{f^{\prime}}$ denote these two intervals, respectively.

- If both $I_{f}$ and $I_{f^{\prime}}$ contain $v$, then we compute the (weighted) distances from $v$ to the two wavefronts. This can be done as follows. Since $v \in I_{f}, v$ must be reached by the leftmost generator $\alpha$ of $W(f, e)$. We compute the distance $d(\alpha, v)$ by computing the tangent from $v$ to $\alpha$ in $O(\log n)$ time. Similarly, we compute $d\left(\alpha^{\prime}, v\right)$, where $\alpha^{\prime}$ is leftmost generator of $W\left(f^{\prime}, e\right)$. If $d(\alpha, v) \leq d\left(\alpha^{\prime}, v\right)$, then the claim of $W(f, e)$ is to the left of that of $W\left(f^{\prime}, e\right)$; otherwise, the claim of $W(f, e)$ is to the right of that of $W\left(f^{\prime}, e\right)$.

- If not both $I_{f}$ and $I_{f^{\prime}}$ contain $v$, then the order of the left endpoints of $I_{f}$ and $I_{f}$, will give the answer.

Without loss of generality, we assume that the claim of $W(f, e)$ is to the left of that of $W\left(f^{\prime}, e\right)$. We next compute the interval $I$ of $e$ claimed by $W(f, e)$ with respect to $W\left(f^{\prime}, e\right)$. Note that the left endpoint of $I$ is the left endpoint of $I_{f}$. Hence, it remains to find the right endpoint of $I$, as follows.

Let $\ell_{e}$ be the supporting line of $e$. Let $\alpha$ be the rightmost generator of $W(f, e)$ and let $\alpha^{\prime}$ be the leftmost generator of $W\left(f^{\prime}, e\right)$. Let $q_{1}$ be the left endpoint of the interval on $e$ claimed by $\alpha$ in $W(f, e)$, i.e., $q_{1}$ is the intersection of the bisector $B\left(\alpha_{1}, \alpha\right)$ and $e$, where $\alpha_{1}$ is the left neighboring generator of $\alpha$ in $W(f, e)$. Similarly, let $q_{2}$ be the right endpoint of the interval on $e$ claimed by $\alpha^{\prime}$ in $W\left(f^{\prime}, e\right)$, i.e., $q_{2}$ is the intersection of $e$ and the bisector $B\left(\alpha^{\prime}, \alpha_{1}^{\prime}\right)$, where $\alpha_{1}^{\prime}$ is the right neighboring generator of $\alpha^{\prime}$ in $W\left(f^{\prime}, e\right)$. Let $q_{0}$ be the intersection of the bisector $B\left(\alpha, \alpha^{\prime}\right)$ and $e$. We assume that the three points $q_{i}, i=0,1,2$ are available and each of them can be computed in $O(\log n)$ time by a bisector-line intersection operation given in Lemma 3.5. If $q_{0}$ is between $q_{1}$ and $q_{2}$, then $q_{0}$ is the right endpoint of $I$ and we can stop the algorithm. If $q_{0}$ is to the left of $q_{1}$, then we delete $\alpha$ from $W(f, e)$. If $q_{0}$ is to the right of $q_{2}$, then we delete $\alpha^{\prime}$ from $W\left(f^{\prime}, e\right)$. In either case, we continue the same algorithm by redefining $\alpha$ or $\alpha^{\prime}$ (and recomputing $q_{i}$ for $i=0,1,2$ ).

Clearly, the above algorithm takes $O((1+k) \log n)$ time, where $k$ is the number of generators that are deleted. We apply the algorithm on $f$ and other $f^{\prime}$ in input $(e)$ to compute the corresponding intervals for $f$. The common intersection of all these intervals is the interval of $e$ claimed by $W(f, e)$. We do so for each $f \in \operatorname{input}(e)$, after which $W(e)$ is obtained. Since the size of input $(e)$ is $O(1)$, we obtain the following lemma.

Lemma 3.4. Given all contributing wavefronts $W(f, e)$ of edges $f \in \operatorname{input}(e)$ for $W(e)$, we can compute the interval of e claimed by each $W(f, e)$ and thus construct $W(e)$ in $O((1+k) \log n)$ time, where $k$ is the total number of generators in all wavefronts $W(f, e)$ that are absent from $W(e)$.

LEMMA 3.5. (Bisector-line intersection) Each bisector-line intersection operation can be performed in $O(\log n)$ time.

\subsection{The Wavefront Propagation Procedure}

In this section, we discuss the wavefront propagation procedure, which is to compute the wavefront $W(e, g)$ for all transparent edges $g \in$ output $(e)$ based on $W(e)$. Consider a transparent edge $g \in$ output $(e)$. The wavefront $W(e, g)$ refers to the portion of $W(e)$ that arrives at $g$ through the well-covering region $\mathcal{U}(g)$ of $g$ if $e \in \operatorname{input}(g)$ and through $\mathcal{U}(e)$ otherwise (in the latter case $g \in \operatorname{input}(e))$. We will need to handle the bisector events, i.e., the intersections between bisectors and the intersections between bisectors and obstacle edges. The HS algorithm processes the bisector events in temporal order, i.e., in order of the simulation time $\tau$. The HSY algorithm instead proposes a simpler approach that processes the events in spatial order, i.e., in order of their geometric locations. We will adapt the HSY's spacial-order method.

Recall that each wavefront $W(e)$ is represented by a list of generators, which are maintained in the leaves of a fully-persistent balanced binary search tree $T(e)$. We further assign each generator a "next bisector event", which is the intersection of its two bounding bisectors. We further endow the tree $T(e)$ with additional node-fields so that each internal node stores a value that is equal to the minimum (resp., maximum) $x$-coordinate (resp., $y$-coordinate) among all bisector events stored at the leaves of the subtree rooted at the node. Using these extra values, we can find from a query range of generators the generator whose bisector event has the minimum/maximum $x$ - or $y$-coordinate in logarithmic time.

The propagation from $W(e)$ to $g$ through $\mathcal{U}$ is done cell by cell, where $\mathcal{U}$ is either $\mathcal{U}(e)$ or $\mathcal{U}(g)$. We start propagating $W(e)$ to the adjacent cell $c$ of $e$ in $\mathcal{U}$ to compute the wavefront through all edges of $c$. Then by using the computed wavefronts on the edges of $c$, we recursively run the algorithm on cells of $\mathcal{U}$ adjacent to $c$. As $\mathcal{U}$ has $O(1)$ cells, the propagation passes through $O(1)$ cells. Hence, the essential ingredient of the algorithm is to propagate a single wavefront, say, $W(e)$, across a single cell $c$ with $e$ on its boundary. Depending on whether $c$ is an empty rectangle, there are two cases.

3.5.1 c Is an Empty Rectangle. We first consider the case where $c$ is an empty rectangle, i.e., there is no island inside $c$ and $c$ does not intersect any obstacle. Without loss of generality, we assume that $e$ is an edge on the bottom side of $c$, and thus all generators of $W(e)$ are below $e$. Our goal is to compute $W(e, g)$, i.e., the generators of $W(e)$ claiming $g$, for all other edges $g$ of $c$. Our algorithm is similar to the HSY algorithm in the high level but the low level implementations are quite different. The main difference is that each bisector in the HSY algorithm is of constant size while this is not the case in our problem. Due to this, it takes constant time to compute the intersection of two bisectors in the HSY algorithm while in our problem this costs $O(\log n)$ time.

The technical crux of the algorithm is to process the intersections in $c$ among the bisectors of generators of $W(e)$. Since all generators of $W(e)$ are below $e$, their bisectors in $c$ are $y$-monotone by Corollary 3.3. This is a critical property our algorithm relies on. Due to the property, we only need to compute $W(e, g)$ for all edges $g$ on the left, right, and top sides of $c$. Another helpful property is that since we propagate $W(e)$ through $e$ inside $c$, if a generator of $\alpha$ of $W(e)$ claims a point $q \in c$, then the tangent from $q$ to $\alpha$ must cross $e$; we refer it as the propagation property. Due to this property, the points of $c$ claimed by $\alpha$ must be to the right of the tangent 
ray from the left endpoint of $e$ to $\alpha$, as well as to the left of the tangent ray from the right endpoint of $e$ to $\alpha$. We call the former ray the left bounding ray of $\alpha$ and the latter the right bounding ray of $\alpha$. As such, for the leftmost generator of $W(e)$, we consider its left bounding ray as its left bounding bisector; similarly, for the rightmost generator of $W(e)$, we consider its right bounding ray as its right bounding bisector.

Starting from $e$, we use a horizontal line segment $\ell$ to sweep $c$ upwards until its top side. At any moment during the algorithm, the algorithm maintains a subset $W(\ell)$ of generators of $W(e)$ for $\ell$ by a balanced binary search tree $T(\ell)$; initially $W(\ell)=W(e)$ and $T(\ell)=T(e)$. Let $\left[x_{1}, x_{2}\right] \times\left[y_{1}, y_{2}\right]$ denote the coordinates of $c$. Using the extra fields on the nodes of the tree $T(\ell)$, we compute a maximal prefix $W_{1}(\ell)$ (resp., $W_{2}(\ell)$ ) of generators of $W(\ell)$ such that the bisector events assigned to all generators in it have $x$-coordinates less than $x_{1}$ (resp., larger than $x_{2}$ ). Let $W_{m}(\ell)$ be the remaining elements of $W(\ell)$. By definition, the first and last generators of $W_{m}(\ell)$ have their bisector events with $x$-coordinates in $\left[x_{1}, x_{2}\right]$. As all bisectors are $y$-monotone in $c$, the lowest bisector intersection in $c$ above $\ell$ must be the "next bisector event" $b$ associated with a generator in $W_{m}(\ell)$, which can be found in $O(\log n)$ time using the tree $T(\ell)$. We advance $\ell$ to the $y$-coordinate of $b$ by removing the generator $\alpha$ associated with the event $b$. Finally, we recompute the next bisector events for the two neighbors of $\alpha$ in $W(\ell)$. Lemma 3.6 below shows that each of these bisector intersections can be computed in $O(\log n)$ time by a bisector-bisector intersection operation, using the technique of Kirkpatrick and Snoeyink [31].

LEMMA 3.6. (Bisector-bisector intersection) Every bisectorbisector intersection operation can be performed in $O(\log n)$ time.

The algorithm finishes once $\ell$ is at the top side of $c$. At this moment, no bisector events of $W(\ell)$ are in $c$. Finally, we run the following wavefront splitting step to split $W(\ell)$ to obtain $W(e, g)$ for all edges $g$ on $\zeta$, where $\zeta$ is the union of the left, top, and right sides of $c$. Our algorithm relies on Lemma 3.7.

LEMmA 3.7. The list of generators of $W(\ell)$ are exactly those in $W(\ell)$ claiming $\zeta$ in order.

Our wavefront splitting step for computing $W(e, g)$ of all edges $g \in \zeta$ works as follows. Consider an edge $g \in \zeta$. Without loss of generality, we assume that the points of $\zeta$ are clockwise around $c$ so that we can talk about their relative order.

Let $p_{l}$ and $p_{r}$ be the front and rear endpoints of $g$, respectively. Let $\alpha_{l}$ and $\alpha_{r}$ be the generators of $W(\ell)$ claiming $p_{l}$ and $p_{r}$, respectively. Then all generators of $W(\ell)$ to the left of $\alpha_{l}$ including $\alpha_{l}$ form the wavefront for all edges of $\zeta$ in the front of $g$; all generators of $W(\ell)$ to the right of $\alpha_{r}$ including $\alpha_{r}$ form the wavefront for all edges of $\zeta$ after $g$; all generators of $W(\ell)$ between $\alpha_{l}$ and $\alpha_{r}$ including $\alpha_{l}$ and $\alpha_{r}$ form $W(e, g)$. Hence, once $\alpha_{l}$ and $\alpha_{r}$ are known, $W(e, g)$ can be obtained by splitting $W(\ell)$ in $O(\log n)$ time. It remains to compute $\alpha_{l}$ and $\alpha_{r}$. Below, we only discuss how to compute the generator $\alpha_{l}$ since $\alpha_{r}$ can be computed analogously.

Starting from the root $v$ of $T(\ell)$, we determine the intersection $q$ between $B\left(\alpha_{1}, \alpha_{2}\right)$ and $\zeta$, where $\alpha_{1}$ is the rightmost generator in the left subtree of $v$ and $\alpha_{2}$ is the leftmost generator of the right subtree of $v$. If $q$ is in the front of $g$ on $\zeta$, then we proceed to the right subtree of $v$; otherwise, we proceed to the left subtree of $v$.
It is easy to see that the runtime of the algorithm is bounded by $O(\eta \cdot \log n)$ time, where $\eta$ is the time for computing $q$. In the HSY algorithm, each bisector is of constant size and an oracle is assumed to exist that can compute $q$ in $O(1)$ time. In our problem, since a bisector may not be of constant size, it is not clear how to bound $\eta$ by $O(1)$. But $\eta$ can be bounded by $O(\log n)$ using the bisector-line intersection operation in Lemma 3.5. Thus, $\alpha_{l}$ can be computed in $O\left(\log ^{2} n\right)$ time. However, this is not sufficient for our purpose, as this would lead to an overall $O\left(n+h \log ^{2} h\right)$ time algorithm. We instead use the following binary search plus bisector tracing approach.

During the wavefront expansion algorithm, for each pair of neighboring generators $\alpha=(A, a)$ and $\alpha^{\prime}=\left(A^{\prime}, a^{\prime}\right)$ in a wavefront (e.g., $W(e))$, we maintain a special point $z\left(\alpha, \alpha^{\prime}\right)$ on the bisector $B\left(\alpha, \alpha^{\prime}\right)$. For example, in the above sweeping algorithm, whenever a generator $\alpha$ is deleted from $W(\ell)$ at a bisector event $b=B\left(\alpha_{l}, \alpha\right) \cap$ $B\left(\alpha, \alpha_{r}\right)$, its two neighbors $\alpha_{l}$ and $\alpha_{r}$ now become neighboring in $W(\ell)$. Then, we initialize $z\left(\alpha_{l}, \alpha_{r}\right)$ to $b$ (the tangent points from $b$ to $\alpha_{l}$ and $\alpha_{r}$ are also associated with $b$ ). During the algorithm, the point $z\left(\alpha_{l}, \alpha_{r}\right)$ will move on $B\left(\alpha, \alpha^{\prime}\right)$ further away from the two defining generators $\alpha$ and $\alpha^{\prime}$ and the movement will trace out the hyperbolic-arcs of the bisector. We call $z\left(\alpha, \alpha^{\prime}\right)$ the tracing-point of $B\left(\alpha, \alpha^{\prime}\right)$. Our algorithm maintains a variant that the tracing point of each bisector of $W(\ell)$ is below the sweeping line $\ell$ (initially, the tracing point of each bisector of $W(e)$ is below $e$ ).

With the above $z$-points, we compute the generator $\alpha_{l}$ as follows. Like the above algorithm, starting from the root $v$ of $T(\ell)$, let $\alpha_{1}$ and $\alpha_{2}$ be the two generators as defined above. To compute the intersection $q$ between $B\left(\alpha_{1}, \alpha_{2}\right)$ and $\zeta$, we trace out the bisector $B\left(\alpha_{1}, \alpha_{2}\right)$ by moving its tracing-point $z\left(\alpha_{1}, \alpha_{2}\right)$ upwards (each time trace out a hyperbolic-arc of $\left.B\left(\alpha_{1}, \alpha_{2}\right)\right)$ until the current tracing hyperbolic-arc intersects $\zeta$ at $q$. If $q$ is in the front of $e$ on $\zeta$, we proceed to the right subtree of $v$; otherwise, we proceed to the left.

After $W(e, g)$ is obtained, we compute $W\left(e, g^{\prime}\right)$ for other edges $g^{\prime}$ on $\zeta$ using the same algorithm as above. For the time analysis, observe that each bisector hyperbolic-arc will be traced out at most once in the wavefront splitting step for all edges of $\zeta$ because the tracing point of each bisector will never move "backwards".

The above propagates $W(e)$ through the cell $c$. Except the final wavefront splitting step, the algorithm runs in $O\left(\left(1+h_{c}\right) \log n\right)$ time, where $h_{c}$ is the number of bisector events in $c$. As $c$ has $O(1)$ edges, the wavefront splitting step takes $O\left(\log n+n_{c}\right)$ time, where $n_{c}$ is the number of hyperbolic-arcs of bisectors that are traced out.

3.5.2 $c$ Is not an Empty Rectangle. If $c$ is not an empty rectangle, $c$ has a square hole inside or/and $\partial c$ contains obstacle edges. Without loss of generality, we assume that $e$ is on the bottom side of $c$.

If $c$ contains a square hole, we partition $c$ into four subcells by cutting $c$ with two lines parallel to $e$, each passing through an edge of the hole. If $c$ has obstacle edges on its boundary, recall that these obstacles edges belong to $O(1)$ convex chains (each of which is a fragment of an elementary chain); we further partition $c$ by additional edges parallel to $e$, so that each resulting subcell contains at most two convex chains, one the left side and the other on the right side. Since $\partial c$ has $O(1)$ convex chains, $O(1)$ additional edges suffices to partition $c$ into $O(1)$ subcells as above. Then, we propagate $e$ through the subcells of $c$, one by one. Below, we describe 
the algorithm for one such subcell. By slightly abusing the notation, we still use $c$ to denote the subcell with $e$ on its bottom side.

Since $\partial c$ has obstacle edges, the propagation algorithm becomes more complicated. As in the HSY algorithm, comparing with the algorithm for the previous case, there are two new bisector events.

- First, a bisector may intersect a convex chain (and thus intersect an obstacle). The HSY algorithm does not explicitly compute these bisector events because such an oracle is not assumed to exist. In our algorithm, however, because the obstacles in our problem are polygonal, we can explicitly determine these events without any special assumption. This is also a reason that the high-level idea of our algorithm is simpler than the HSY algorithm.

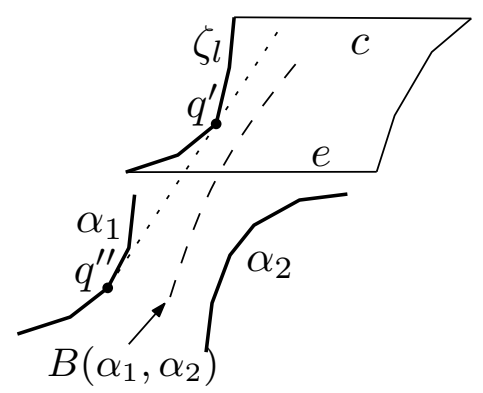

Figure 10: Illustrating the creation of a new generator at $q^{\prime}$.

- Second, new generators may be created at the convex chains. We still sweep a horizontal line $\ell$ from $e$ upwards. Let $W(\ell)$ be the current wavefront at some moment during the algorithm. Consider two neighboring generators $\alpha_{1}$ and $\alpha_{2}$ in $W(\ell)$ with $\alpha_{1}$ on the left of $\alpha_{2}$. We use $\zeta_{l}$ to denote the convex chain on the left side of $c$. Let $q^{\prime}$ be the tangent point on $\zeta_{l}$ of the common tangent between $\zeta_{l}$ and $\alpha_{1}$ and let $q^{\prime \prime}$ be the tangent point on $\alpha_{1}$ (e.g., see Fig. 10). If $d\left(\alpha_{1}, q^{\prime}\right)<d\left(\alpha_{2}, q^{\prime}\right)$, then a new generator $\alpha$ on $\zeta_{l}$ with initial vertex $q^{\prime}$ and weight equal to $d\left(\alpha_{1}, q^{\prime}\right)$ is created (designated counterclockwise direction) and inserted into $W(\ell)$ right before $\alpha_{1}$. The bisector $B\left(\alpha, \alpha_{1}\right)$ is the ray emanating from $q^{\prime}$ and extending away from $q^{\prime \prime}$. The region to the left of the ray has $\alpha$ as its predecessor. When the sweeping line $\ell$ is at $q^{\prime}$, all wavelets in $W(\ell)$ to the left of $\alpha_{1}$ have already collided with $\zeta_{l}$ and thus the first three generators of $W(\ell)$ are $\alpha, \alpha_{1}$, and $\alpha_{2}$.

In what follows, we describe our sweeping algorithm to propagate $W(e)$ through $c$. We begin with an easier case where only the left side of $c$ is a convex chain, denoted by $\zeta_{l}$ (and the right side is a vertical transparent edge, denoted by $\zeta_{r}$ ). We use $\zeta_{t}$ to denote the top side of $c$, which is a transparent edge. As in the previous case, we sweep a line $\ell$ from $e$ upwards until the top side $\zeta_{t}$. During the algorithm, we maintain a list $W(\ell)$ of generators by a balanced binary search tree $T(\ell)$. Initially, $W(\ell)=W(e)$ and $T(\ell)=T(e)$.

We compute the intersection $q$ of the convex chain $\zeta_{l}$ and the bisector $B\left(\alpha_{1}, \alpha_{2}\right)$, for the leftmost bisectors of $\alpha_{1}$ and $\alpha_{2}$ of $W(\ell)$. We call it the bisector-chain intersection operation, which can be performed in $O(\log n)$ time by the following lemma.

LEMMA 3.8. (Bisector-chain intersection) Each bisector-chain intersection operation can be performed in $O(\log n)$ time.
If the intersection $q$ of $\zeta_{l}$ and $B\left(\alpha_{1}, \alpha_{2}\right)$ does not exist, then we compute the tangent between $\zeta_{l}$ and $\alpha_{1}$, which can be done in $O(\log n)$ time [38]; let $q^{\prime}$ be the tangent point at $\zeta_{l}$. Regardless whether $q$ exists or not, we compute the lowest bisector intersection $b$ in $c$ above $\ell$ in the same way as in the algorithm for the previous case where $c$ is an empty rectangle. Depending on whether $q$ exists or not, we proceed as follows. For any point $p$ in the plane, let $y(p)$ denote the $y$-coordinate of $p$.

(1) If $q$ exists, then depending on whether $y(q) \leq y(b)$, there are two subcases. If $y(q) \leq y(b)$, then we process the bisector event $q$ : remove $\alpha_{1}$ from $W(\ell)$ and then recompute $q, q^{\prime}$, and $b$. Otherwise, we process the bisector event at $b$ in the same way as in the previous case and then recompute $q, q^{\prime}$, and $b$.

(2) If $q$ does not exist, then depending on whether $y(b) \leq y\left(q^{\prime}\right)$, there are two subcases. If $y(b) \leq y\left(q^{\prime}\right)$, then we process the bisector event at $b$ in the same way as before and then recompute $q, q^{\prime}$, and $b$. Otherwise, we insert a new generator $\alpha=\left(A, q^{\prime}\right)$ to $W(\ell)$ as the leftmost generator, where $A$ is the fragment of the elementary chain containing $\zeta_{l}$ from $q^{\prime}$ counterclockwise to the end of the chain, and $\alpha$ is designated the counterclockwise direction of $A$ and the weight of $q^{\prime}$ is $d\left(\alpha_{1}, q^{\prime}\right)$; e.g., see Fig. 10 . The ray from $q^{\prime}$ in the direction from $q^{\prime \prime}$ to $q^{\prime}$ is the bisector of $\alpha$ and $\alpha_{1}$, where $q^{\prime \prime}$ is the tangent point on $\alpha_{1}$ of the common tangent between $\alpha_{1}$ and $\zeta_{l}$. We initialize the tracing-point $z\left(\alpha, \alpha_{1}\right)$ of $B\left(\alpha, \alpha_{1}\right)$ to $q^{\prime}$. Finally, we recompute $q, q^{\prime}$, and $b$.

Once the sweep line $\ell$ reaches the top side $\zeta_{t}$ of $c$, the algorithm stops. Finally, we run a wavefront splitting step as before. Because the left side $\zeta_{l}$ consists of obstacle edges, we split $W(\ell)$ to compute $W(e, g)$ for all transparent edges $g$ on the top side $\zeta_{t}$ and the right side $\zeta_{r}$ of $c$. The algorithm is the same as the previous case.

The above discusses the case where only the left side $\zeta_{l}$ of $c$ is a convex chain. For the general case where both the left and right sides of $c$ are convex chains, the algorithm is similar. The difference is that we have to compute a point $p$ corresponding to $q$ and a point $p^{\prime}$ corresponding to $q^{\prime}$ on the right side $\zeta_{r}$ of $c$. Specifically, $p$ is the intersection of $B\left(\alpha_{2}^{\prime}, \alpha_{1}^{\prime}\right)$ with $\zeta_{r}$, where $\alpha_{2}^{\prime}$ and $\alpha_{1}^{\prime}$ are the two rightmost generators of $W$. If $p$ does not exist, we compute the common tangent of $\zeta_{r}$ and $\alpha_{1}^{\prime}$, and $p^{\prime}$ is the tangent point on $\zeta_{r}$.

If $q$ does not exist, we let $y(q)$ be $\infty$; otherwise, $q^{\prime}$ is not needed and we let $y\left(q^{\prime}\right)$ be $\infty$. We apply the same convention to $p$ and $p^{\prime}$. We define $b$ as the bisector event in the same way as before. In each step, we process the lowest point $r$ of $\left\{q, q^{\prime}, b, p, p^{\prime}\right\}$. If $r$ is $q$ or $p$, we process it in the same way as before for $q$. If $r$ is $q^{\prime}$ or $p^{\prime}$, we process it in the same way as before for $q^{\prime}$. If $r$ is $b$, we process it in the same way as before. After processing $r$, we recompute the five points. Each step takes $O(\log n)$ time. After the sweep line $\ell$ reaches the top side $\zeta_{t}$ of $c, W(\ell)$ is $W\left(e, \zeta_{t}\right)$ for the top side $\zeta_{t}$ of $c$ because both the left and right sides of $c$ are obstacle edges. Finally, we run the wavefront splitting step on $W(\ell)$ to compute the wavefronts $W(e, g)$ for all transparent edges $g$ on $\zeta_{t}$.

In summary, propagating $W(e)$ through $c$ takes $O\left(\left(1+h_{c}\right) \cdot \log n+\right.$ $n_{c}$ ) time, where $h_{c}$ is the number of bisector events (including both the bisector-bisector intersection events and the bisector-obstacle intersection events) and $n_{c}$ is the number of hyperbolic-arcs of bisectors that are traced out in the wavefront splitting step. 
We use the following lemma to summarize the algorithm for both cases (i.e., regardless whether $c$ is an empty rectangle or not).

Lemma 3.9. Suppose $W(e)$ is a wavefront on a transparent edge of a cell $c$ of the subdivision $\mathcal{S}^{\prime}$. Then, $W(e)$ can be propagated through $c$ to all other transparent edges of $c$ in $O\left(\left(1+h_{c}\right) \log n+n_{c}\right)$ time, where $h_{c}$ is the number of bisector events and $n_{c}$ is the number of hyperbolicarcs of bisectors that are traced out in the wavefront splitting step.

\subsection{Time Analysis}

We show that the wavefront expansion algorithm described above runs in $O(n+h \log h)$ time and space. For this and also for the purpose of constructing the shortest path map $S P M^{\prime}(s)$ later in Section 3.7, as in the HS algorithm, we mark generators in the way that if a generator $\alpha$ is involved in a true bisector event of $S P M(s)$ (either a bisector-bisector intersection or a bisector-obstacle intersection) in a cell $c$ of the subdivision $\mathcal{S}^{\prime}$, then $\alpha$ is guaranteed to be in a set of marked generators for $c$. The generator marking rules, which are omitted, are consistent with those in the HS algorithm.

LEMma 3.10. The total number of marked generators during the algorithm is at most $O(h)$.

Next we use Lemma 3.10 to analyze the time of the algorithm. First of all, by Lemma 3.1, constructing the conforming subdivision $\mathcal{S}^{\prime}$ can be done in $O(n+h \log h)$ time and $O(n)$ space. The wavefront expansion algorithm has two main subroutines: the wavefront merging procedure and the wavefront propagation procedure.

For the wavefront merging procedure, by Lemma 3.4, this step takes $O((1+k) \log n)$ time, where $k$ is the total number of generators in all wavefronts $W(f, e)$ that are absent from $W(e)$. According to the algorithm, if a generator $\alpha$ is absent from $W(e)$, it must be deleted at a bisector event. Thus $\alpha$ must be marked by our marking rules. Due to Lemma 3.10, the total sum of $k$ in the entire algorithm is $O(h)$. As such, the wavefront merging procedure in the entire algorithm takes $O(h \log n)$ time in total.

By Lemma 3.9, the wavefront propagation procedure runs in $O\left(\left(1+h_{c}\right) \log n+n_{c}\right)$ time, where $h_{c}$ is the number of bisector events and $n_{c}$ is the number of hyperbolic-arcs of bisectors that are traced out in the wavefront splitting step. For each bisector-bisector intersection event, at least one involved generator is marked by Rule 3(b). For each bisector-obstacle intersection event, at least one involved generator is marked by our marking rules. Hence, by Lemma 3.10, the total sum of $h_{c}$ in the entire algorithm is $O(h)$. In addition, Lemma 3.11 below shows that the total sum of $n_{c}$ in the entire algorithm is $O(n)$. Therefore, the wavefront propagation procedure in the entire algorithm takes $O(n+h \log n)$ time in total.

LEMмA 3.11. The total number of traced hyperbolic-arcs of the bisectors in the entire algorithm is $O(n)$.

In summary, the total time of the wavefront expansion algorithm is $O(n+h \log n)$, which is $O(n+h \log h)$.

For the space complexity of the algorithm, since each wavefront $W(e)$ is maintained by a persistent binary tree, each bisector event costs additional $O(\log n)$ space. As discussed above, the total sum of $k+h_{c}$ in the entire algorithm, which is the total number of bisector events, is $O(h)$. Hence, the space cost by persistent binary trees is $O(h \log n)$. The space used by other parts of the algorithm is $O(n)$. Hence, the space complexity of the algorithm is $O(n+h \log h)$.

\subsection{Computing the Shortest Path Map $\operatorname{SPM}(s)$}

The map $\operatorname{SPM}(s)$ can be constructed in $O(n+h \log h)$ time using the marked generators. As discussed before, it suffices to construct $S P M^{\prime}(s)$. We first have the following lemma.

Lemma 3.12. If a generator $\alpha$ participates in a bisector event of $S P M^{\prime}(s)$ in a cell $c$ of $\mathcal{S}^{\prime}$, then $\alpha$ must be marked in $c$.

To construct $S P M^{\prime}(s)$, with the help of Lemma 3.12, the highlevel algorithmic scheme is similar to the HS algorithm although many details are different. There are two main steps. The first main step is to identify all vertices of $S P M^{\prime}(s)$ and the second one is to compute the edges of $S P M^{\prime}(s)$ and assemble them to obtain $S P M^{\prime}(s)$. We omit all details here. In summary, $S P M^{\prime}(s)$ can be computed in $O(n+h \log h)$ time. As discussed before, the shortest path map $\operatorname{SPM}(s)$ can be built in additional $O(n)$ time.

\subsection{Reducing the Space to $O(n)$}

The algorithm above computes SPM(s) in $O(n+h \log h)$ time and $O(n+h \log h)$ space. We now reduce to the space to $O(n)$, using the technique given in [44]. The reason that the above algorithm needs $O(n+h \log h)$ space is two-fold. First, it uses fully persistent binary trees (with path-copying) to represent wavefronts $W(e)$. Because there are $O(h)$ bisector events in the wavefront expansion algorithm and each event costs $O(\log n)$ additional space on a persistent tree, the total space of the algorithm is $O(n+h \log n)$. Second, in order to construct $S P M(s)$ after the wavefront expansion algorithm, the wavefronts $W(e)$ of all transparent edges $e$ of $\mathcal{S}^{\prime}$ are needed in the algorithm of Section 3.7, and the wavefronts $W(e)$ are maintained in those persistent trees. We resolve these two issues below.

3.8.1 Reducing the Space of the Wavefront Expansion Algorithm. We still use persistent trees to represent wavefronts. However, as there are $O(h)$ bisector events in total in the algorithm, we divide the algorithm into $O(\log h)$ phases so that each phase has no more than $h / \log h$ events. The total additional space for processing the events using persistent trees in each phase is $O(h)$. At the end of each phase, we "reset" the space of the algorithm by only storing a "snapshot" of the algorithm (and discarding all other used space) so that (1) the snapshot contains sufficient information for the subsequent algorithm to proceed as usual, and (2) the total space of the snapshot is $O(h)$.

Specifically, we make the following changes to the wavefront propagation procedure, which is to compute the wavefronts $W(e, g)$ for all edges $g \in$ output(e) using the wavefront $W(e)$. We now maintain a counter count to record the number of bisector events that have been processed so far since the last space reset; count = 0 initially. Consider a wavefront propagation procedure on the wavefront $W(e)$ of a transparent edge $e$. The algorithm will compute $W(e, g)$ for all edges $g \in$ output $(e)$, by propagating $W(e)$. We apply the same algorithm as before. For each bisector event, we first do the same as before. Then, we increment count by one. If count $<h / \log h$, we proceed as before (i.e., process the next event). Otherwise, we have reached the end of the current phase and will start a new phase. To do so, we first reset count $=0$ and then reset the space by constructing and storing a snapshot of the algorithm (other space occupied by the algorithm is discarded), as follows. 
(1) Let $g$ refer to the edge of output(e) whose $W(e, g)$ is currently being computed in the algorithm. We store the tree that is currently being used to compute $W(e, g)$ right after the above event. To do so, we can make a new tree by copying the newest version of the current persistent tree the algorithm is operating on. The size of the tree is bounded by $O(h)$. We will use this tree to "resume" computing $W(e, g)$ in the subsequent algorithm.

(2) For each $g^{\prime} \in$ output $(e) \backslash\{g\}$ whose $W\left(e, g^{\prime}\right)$ has been computed, we store the tree for $W\left(e, g^{\prime}\right)$, which will be used to to compute the wavefronts $W\left(g^{\prime}\right)$ of $g^{\prime}$ in the later algorithm.

(3) We store the tree for the wavefront $W(e)$. Note that the tree may have many versions due to processing the events and we only keep its original version for $W(e)$. Hence, the size of the tree is $O(h)$. This tree will be used in the subsequent algorithm to compute $W\left(e, g^{\prime}\right)$ for those edges $g^{\prime} \in$ output $(e) \backslash\{g\}$ whose $W\left(e, g^{\prime}\right)$ have not been computed yet.

(4) We check every transparent edge $e^{\prime}$ of $\mathcal{S}^{\prime}$ with $e^{\prime} \neq e$. If $e^{\prime}$ has been processed (i.e., the wavefront propagation procedure has been called on $\left.W\left(e^{\prime}\right)\right)$ and there is an edge $g^{\prime} \in \operatorname{output}\left(e^{\prime}\right)$ that has not been processed, we know that $W\left(e^{\prime}, g^{\prime}\right)$ has been computed and is available; we store the tree for $W\left(e^{\prime}, g^{\prime}\right)$. We will use the tree to compute the wavefronts $W\left(g^{\prime}\right)$ of $g^{\prime}$ in the subsequent algorithm.

We call the wavefronts stored above the snapshot; intuitively, it contains all wavelets in the forefront of the wavefont expansion. By the same analysis as in [44], we can show that the snapshot contains sufficient information for the subsequent algorithm to proceed as usual and the total space of the snapshot is $O(h)$.

The above discusses our changes to the wavefront propagation procedure. For the merging procedure, which is to construct $W(e)$ from $W(f, e)$ for the edges $f \in \operatorname{input}(e)$, notice that we do not need the old versions of $W(f, e)$ anymore after $W(e)$ is constructed. Therefore, it is not necessary to use the path-copying method to process each event in the procedure. Hence, the total space needed in the wavefront merging procedure in the entire algorithm is $O(n)$.

3.8.2 Reducing the Space of Constructing $\operatorname{SPM}^{\prime}(s)$. For the second issue of constructing $S_{P M}(s)$, the algorithm in Section 3.7 relies on the wavefronts $W(e)$ for all transparent edges $e$, which are maintained by persistent trees. Due to the space-reset, the wavefronts are not maintained anymore, and thus we need to restore these wavefronts in order to construct $S P M^{\prime}(s)$. To this end, a key observation is that by marking a total of $O(h)$ additional wavelet generators it is possible to restore all historical wavefronts that are needed for constructing $S P M^{\prime}(s)$. In this way, $S P M^{\prime}(s)$ can be constructed in $O(n+h \log h)$ time and $O(n)$ space. The details are omitted.

\section{THE GENERAL CASE}

In this section, we extend our convex case algorithm in Section 3 to the general case where obstacles of $\mathcal{P}$ may not be convex. We use an extended corridor structure of $\mathcal{P}$, which has been used to solve various problems in polygonal domains [4, 7-9, 27-29, 37].

\subsection{The Extended Corridor Structure}

The structure partitions the free space $\mathcal{F}$ into an ocean $\mathcal{M}, O(n)$ bays, and $O(h)$ canals. Each bay is a simple polygon that shares an edge with $\mathcal{M}$. Each canal is a simple polygon that shares two edges with $\mathcal{M}$. But two bays or two canals, or a bay and a canal do not share any edge. A common edge of a bay (or canal) with $\mathcal{M}$ is called a gate (which is in the interior of $\mathcal{F}$ except its two endpoints). Thus each bay has one gate and each canal has two gates. Further, $\mathcal{M}$ is bounded by $O(h)$ convex chains. After $\mathcal{P}$ is triangulated, $\mathcal{M}$ and all bays and canals can be obtained in $O(n)$ time [29]. We omit the detailed definitions of the ocean, bays, and canals, but instead only use Fig. 11 to illustrate a bay bay $(\overline{c d})$ and a canal canal $(x, y)$.
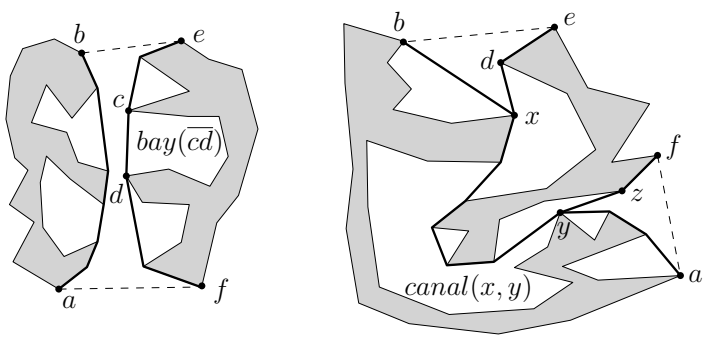

Figure 11: Illustrating a bay $b a y(\overline{c d})$ with gate $\overline{c d}$ (left) and a canal $\operatorname{canal}(x, y)$ with gates $\overline{x d}$ and $\overline{y z}$ (right). The thick path between $x$ and $y$ is the corridor path of $\operatorname{canal}(x, y)$.

A useful property is that if both $s$ and $t$ are in $\mathcal{M}$, then any shortest $s-t$ path must be in the union of $\mathcal{M}$ and all corridor paths, each of which is contained in a canal. In light of this property, we propose the following algorithm. Let $s$ be a given source point. By considering $s$ as a special obstacle of $\mathcal{P}$, we construct the extended corridor structure of $\mathcal{P}$. Consider any query point $t$, which may be in the ocean $\mathcal{M}$, a bay bay $(\overline{c d})$, or a canal canal $(x, y)$.

- If $t \in \mathcal{M}$, then the union of $\mathcal{M}$ and all corridor paths contains a shortest $s$ - $t$ path. To handle this case, we will build a shortest path map $\operatorname{SPM}(\mathcal{M})$ in $\mathcal{M}$ with respect to the union of $\mathcal{M}$ and all corridor paths. In face, $\operatorname{SPM}(\mathcal{M})$ is exactly the portion of $\operatorname{SPM}(s)$ in $\mathcal{M}$, i.e., $\operatorname{SPM}(s) \cap \mathcal{M}$. To build $\operatorname{SPM}(\mathcal{M})$, a key observation is that the boundary $\partial \mathcal{M}$ consists of $O(h)$ convex chains. Therefore, we can easily adapt our previous algorithm for the convex case. However, the algorithm needs to be modified so that the corridor paths should be taken into consideration. Intuitively, corridor paths provide certain kind of "shortcuts" for wavefronts to propagate.

- If $t$ is in a bay bay $(\overline{c d})$, then any shortest $s$ - $t$ path must cross its gate $\overline{c d}$. To handle this case, we will extend $\operatorname{SPM}(\mathcal{M})$ into bay $(\overline{c d})$ through the gate $\overline{c d}$ to construct the shortest path map in $b a y(\overline{c d})$, i.e., the portion of $\operatorname{SPM}(s)$ in $b a y(\overline{c d})$, $\operatorname{SPM}(s) \cap \operatorname{bay}(\overline{c d})$.

- If $t$ is in a canal $\operatorname{canal}(x, y)$, then any shortest $s$ - $t$ path must cross one of the two gates of the canal. To handle this case, we will extend $\operatorname{SPM}(\mathcal{M})$ into $\operatorname{canal}(x, y)$ through the two gates to construct the shortest path map in $\operatorname{canal}(x, y)$, i.e., the portion of $\operatorname{SPM}(s)$ in $\operatorname{canal}(x, y), \operatorname{SPM}(s) \cap \operatorname{canal}(x, y)$.

We first describe our algorithm for constructing $\operatorname{SPM}(\mathcal{M})$ in Section 4.2. We then expand $\operatorname{SPM}(\mathcal{M})$ into all bays in Section 4.3 and expand $\operatorname{SPM}(\mathcal{M})$ into all canals in Section 4.4. The algorithm for the canal case utilizes the bay case algorithm as a subroutine. 


\subsection{Constructing the Map in $\operatorname{SPM}(\mathcal{M})$}

As $\partial \mathcal{M}$ consists of $O(h)$ convex chains, we can apply and slightly modify our algorithm for the convex case. To do so, for each convex chain of $\partial \mathcal{M}$, we define its rectilinear extreme vertices in the same way as before. Let $\mathcal{V}$ be the set of the rectilinear extreme vertices of all convex chains. Hence, $|\mathcal{V}|=O(h)$. In addition, to incorporate the corridor paths into the algorithm, we include the endpoints of each corridor path in $\mathcal{V}$. As there are $O(h)$ corridor paths, the size of $\mathcal{V}$ is still bounded by $O(h)$. Note that each corridor path endpoint is also an endpoint of a convex chain of $\partial \mathcal{M}$. We construct the conforming subdivision $\mathcal{S}$ based on the points of $\mathcal{V}$ and then insert the convex chains of $\mathcal{M}$ into $\mathcal{S}$ to obtain $\mathcal{S}^{\prime}$. The algorithm is essentially the same as before. In addition, we make the following changes to $\mathcal{S}^{\prime}$, which is mainly for incorporating the corridor paths into our wavefront expansion algorithm, as will be clear later.

Let $v$ be an endpoint of a corridor path $\pi$. Since $v$ is in $\mathcal{V}, v$ is incident to $O(1)$ transparent edges in $\mathcal{S}^{\prime}$. For each such transparent edge $e$, if $|\pi|<2 \cdot|e|$, then we divide $e$ into two sub-edges such that the length of the one incident to $v$ is equal to $|\pi| / 2$; for each sub-edge, we set its well-covering region the same as $\mathcal{U}(e)$. Note that this does not affect the properties of $\mathcal{S}^{\prime}$. In particular, each transparent edge $e$ is still well-covered. This change guarantees the following property: for each corridor path $\pi,|\pi| \geq 2 \cdot\left|e^{\prime}\right|$, where $e^{\prime}$ is any transparent edge of $\mathcal{S}^{\prime}$ incident to either endpoint of $\pi$. For reference purpose, we refer to it as the corridor path length property.

Next we apply the wavefront expansion algorithm. Here we need to incorporate the corridor paths into the algorithm. Intuitively, each corridor path provides a "shortcut" for the wavefront, i.e., if a wavelet hits an endpoint of a corridor path, then the wavelet will come out of the corridor path from its other endpoint but with a delay of distance equal to the length of the corridor path. More details are given below.

Since all corridor path endpoints are in $\mathcal{V}$, they are vertices of transparent edges of $\mathcal{S}^{\prime}$. Consider an endpoint $v$ of a corridor path $\pi$. Let $u$ be the other endpoint of $\pi$. Recall that the wavefront propagation procedure for $W(e)$ is to propagate $W(e)$ to compute $W(e, g)$ for all edges $g \in$ output $(e)$. In addition to the previous algorithm for the procedure, we also propagate $W(e)$ through the corridor path $\pi$ to $u$. This is done as follows. Recall that when $e$ is processed, since $v$ is an endpoint of $e$, the weighted distance of $v$ through $W(e)$ is equal to $d(s, v)$. Hence, the wavefront $W(e)$ hits $u$ through $\pi$ at time $d(s, v)+|\pi|$. We then update covertime $(g)=$ $\min \{$ covertime $(g), d(s, v)+|\pi|+|g|\}$, for each transparent edge $g$ incident to $u$. We also set the wavefront $W(e, g)$ consisting of the only wavelet with $u$ as the generator with weight equal to $d(s, v)+|\pi|$. Since there are $O(1)$ transparent edges $g$ incident to $u$, the above additional step takes $O(1)$ time, which does not change the time complexity of the overall algorithm asymptotically. The corridor path length property assures that if $W(e)$ contributes to a wavefront $W(g)$ at $g$, then $e$ must be processed earlier than $g$. This guarantees the correctness of the algorithm.

In this way, we can first construct a decomposition $S P M^{\prime}(\mathcal{M})$ of $\mathcal{M}$ in $O(n+h \log h)$ time and $O(n)$ space, where $S P M^{\prime}(\mathcal{M})$ is defined similarly as $S P M^{\prime}(s)$ in Section 3. Then, by a similar algorithm as before for constructing $\operatorname{SPM}(s), \operatorname{SPM}(\mathcal{M})$ can be obtained from $S P M^{\prime}(\mathcal{M})$ in additional $O(n)$ time.

\subsection{Expanding $\operatorname{SPM}(\mathcal{M})$ into Bays}

We now expand $\operatorname{SPM}(\mathcal{M})$ into all bays in $O(n+h \log h)$ time and $O(n)$ space. In fact, we expand $S^{\prime} M^{\prime}(\mathcal{M})$ to the bays. We process each bay individually. Consider a bay bay $(\overline{c d})$ with gate $\overline{c d}$. Without loss of generality, we assume that $\overline{c d}$ is horizontal, $c$ is to the left of $d$, and $b a y(\overline{c d})$ is locally above $\overline{c d}$.

Let $v_{1}, v_{2}, \ldots, v_{m}$ be the vertices of $S P M^{\prime}(\mathcal{M})$ on $\overline{c d}$ ordered from left to right (e.g., see Fig. 12). Let $c=v_{0}$ and $d=v_{m+1}$. Hence, each $\overline{v_{i} v_{i+1}}$ is claimed by a generator $\alpha_{i}=\left(A_{i}, a_{i}\right)$ for all $i=0,1, \ldots, m$. Let $b_{i}$ and $c_{i}$ be the tangent points on $A_{i-1}$ and $A_{i}$ from $v_{i}$, respectively, for each $i=1,2, \ldots, m$ (e.g., see Fig. 12). For $v_{0}$, only $c_{0}$ is defined; for $v_{m+1}$, only $b_{m+1}$ is defined. Observe that for any point $p \in \overline{v_{i} v_{i+1}}$, which is claimed by $\alpha_{i}$, its tangent point on $A_{i}$ must be on the portion of $A_{i}$ between $c_{i}$ and $b_{i+1}$ and we use $A_{i}^{\prime}$ to denote that portion. So with respect to $b a y(\overline{c d})$, we use $\alpha_{i}^{\prime}=\left(A_{i}^{\prime}, a_{i}^{\prime}\right)$ to refer to the generator, where $a_{i}^{\prime}$ refers to the one of $c_{i}$ and $b_{i+1}$ that is closer to $a_{i}$. Hence, for any point $t \in b a y(\overline{c d})$, any shortest path $\pi(s, t)$ from $s$ to $t$ must be via one of the generators $\alpha_{i}^{\prime}$ for $i=0,1, \ldots, m$. Consider the region $R$ bounded by $A_{i}^{\prime}$ for all $i \in[0, m]$, the tangents from $v_{i}$ to their generators for all $i \in[0, m+1]$, and the boundary of the bay excluding its gate. Notice that $R$ is a simple polygon. For any point $t \in \operatorname{bay}(\overline{c d})$, the above observation implies any shortest $s$ - $t$ path $\pi(s, t)$ is the concatenation of a shortest path from $s$ to a generator initial vertex $a_{i}^{\prime}$ and the shortest path from $a_{i}^{\prime}$ to $t$ in $R$.

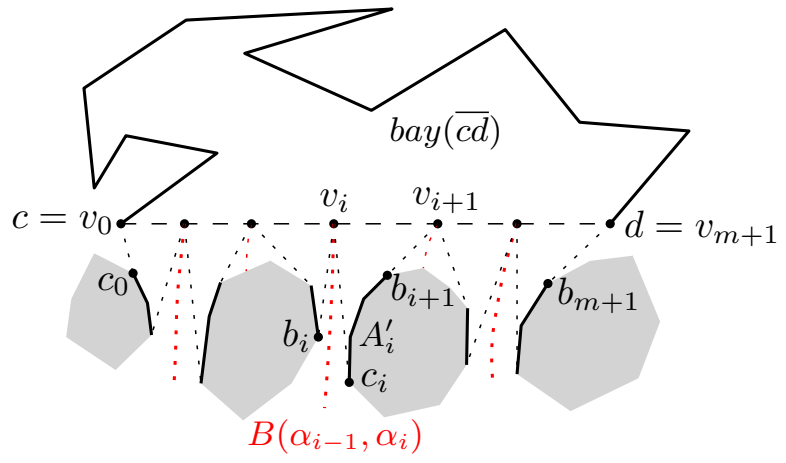

Figure 12: Illustrating $b a y(\overline{c d})$ and the generators. The thick segments on obstacles are $A_{i}^{\prime}, i=0,1, \ldots, m$.

According to the above discussion, expanding $S P M^{\prime}(\mathcal{M})$ into $b a y(\overline{c d})$ is equivalent to the following weighted geodesic Voronoi diagram problem in a simple polygon: Partition $R$ into cells with respect to the point sites $a_{0}^{\prime}, a_{1}^{\prime}, \ldots, a_{m}^{\prime}$ (with weights equal to their geodesic distances to $s$ ) such that all points in the same cell have the same closest site. Let $n_{b}$ be the number of vertices in $b a y(\overline{c d})$ (the subscript "b" represents "bay"). Let $n_{g}$ be the total number of obstacle vertices in $A_{i}^{\prime}$ for all $i \in[0, m]$ (the subscript "g" represents "generator"). Note that $v_{i}$ for all $i=1, \ldots, m$ are also vertices of $R$. Hence, the number of vertices of $R$ is $n_{b}+n_{g}+m$. We can solve the above problem in $O\left(m \log m+n_{b}+n_{g}\right)$ and $O\left(n_{b}+n_{g}+m\right)$ space.

We now analyze the total time for processing all bays. Since $S P M^{\prime}(\mathcal{M})$ has $O(h)$ vertices, the total sum of $m$ for all bays is $O(h)$. The total sum of $n_{b}$ for all bays is at most $n$. Notice that the obstacle 
edges on $A_{i}^{\prime}$ are disjoint for different bays, and thus the total sum of $n_{g}$ for all bays is $O(n)$. Hence, expanding $\operatorname{SPM}^{\prime}(\mathcal{M})$ to all bays takes $O(n+h \log h)$ time and $O(n)$ space in total.

\subsection{Expanding $S P M^{\prime}(\mathcal{M})$ into Canals}

Consider a canal $\operatorname{canal}(x, y)$ with two gates $\overline{x d}$ and $\overline{y z}$. The goal is to expand the map $\operatorname{SPM}^{\prime}(\mathcal{M})$ into $\operatorname{canal}(x, y)$ through the two gates to obtain the shortest path map in the canal, denoted by $\operatorname{SPM}(\operatorname{canal}(x, y))$. The high-level scheme of the algorithm is similar in spirit to that for the $L_{1}$ problem [9]. The algorithm has three main steps. First, we expand $S P M^{\prime}(\mathcal{M})$ into $\operatorname{canal}(x, y)$ through the gate $\overline{x d}$, by applying our algorithm for bays. Let $S P M_{1}(\operatorname{canal}(x, y)) \mathrm{de}-$ note the map of $\operatorname{canal}(x, y)$ obtained by the algorithm. Second, we expand $\operatorname{SPM}(\mathcal{M})$ into canal $(x, y)$ through the gate $\overline{y z}$ by a similar algorithm as above; let $\operatorname{SPM}_{2}(\operatorname{canal}(x, y))$ denote the map of canal $(x, y)$ obtained by the algorithm. Third, we merge the maps $\operatorname{SPM}_{1}(\operatorname{canal}(x, y))$ and $\operatorname{SPM}_{2}(\operatorname{canal}(x, y))$ to obtain the map $\operatorname{SPM}(\operatorname{canal}(x, y))$. This is done by using the merge step from the standard divide-and-conquer Voronoi diagram algorithm to compute the region closer to the generators at $\overline{x d}$ than those at $\overline{y z}$.

The first step takes $O(n+h \log h)$ time for all canals. So is the second step. The third step can be done in linear time in the total size of $\operatorname{SPM}_{1}(\operatorname{canal}(x, y))$ and $\operatorname{SPM}_{2}(\operatorname{canal}(x, y))$. Since the total size of the two maps over all canals is $O(n)$, the total time of the third step for all canals is $O(n)$. Hence, the time for computing the shortest path maps in all canals is $O(n+h \log h)$ and the space is $O(n)$.

Theorem 4.1. Suppose $\mathcal{P}$ is a set of h pairwise disjoint polygonal obstacles with a total of $n$ vertices in the plane and $s$ is a source point. Assume that a triangulation of the free space is given. The shortest path map SPM(s) with respect to san be constructed in $O(n+h \log h)$ time and $O(n)$ space.

With $\operatorname{SPM}(s)$, by building a point location data structure [16, 30] on $\operatorname{SPM}(s)$ in $O(n)$ time, given a query point $t, d(s, t)$ can be computed in $O(\log n)$ time and a shortest $s-t$ path can be produced in additional time linear in the number of edges of the path.

\section{REFERENCES}

[1] S.W. Bae and H. Wang. 2019. $L_{1}$ shortest path queries in simple polygons. Theoretical Computer Science 790 (2019), 105-116.

[2] R. Bar-Yehuda and B. Chazelle. 1994. Triangulating disjoint Jordan chains. International fournal of Computational Geometry and Applications 4, 4 (1994), 475-481.

[3] D.Z. Chen, J. Hershberger, and H. Wang. 2013. Computing shortest paths amid convex pseudodisks. SIAM J. Comput. 42, 3 (2013), 1158-1184.

[4] D.Z. Chen, R. Inkulu, and H. Wang. 2016. Two-point $L_{1}$ shortest path queries in the plane. Fournal of Computational Geometry 1 (2016), 473-519.

[5] D.Z. Chen, K.S. Klenk, and H.-Y.T. Tu. 2000. Shortest path queries among weighted obstacles in the rectilinear plane. SIAM f. Comput. 29, 4 (2000), 1223-1246.

[6] D.Z. Chen and H. Wang. 2015. Computing shortest paths among curved obstacles in the plane. ACM Transactions on Algorithms 11 (2015). Article No. 26.

[7] D.Z. Chen and H. Wang. 2015. A new algorithm for computing visibility graphs of polygonal obstacles in the plane. Fournal of Computational Geometry 6 (2015), 316-345.

[8] D.Z. Chen and H. Wang. 2017. Computing the visibility polygon of an island in a polygonal domain. Algorithmica 77 (2017), 40-64.

[9] D.Z. Chen and H. Wang. 2019. Computing $L_{1}$ shortest paths among polygonal obstacles in the plane. Algorithmica 81 (2019), 2430-2483.

[10] Y.-J. Chiang and J.S.B. Mitchell. 1999. Two-point Euclidean shortest path queries in the plane. In Proceedings of the Annual ACM-SIAM Symposium on Discrete Algorithms (SODA). 215-224.

[11] K.L. Clarkson, R. Cole, and R.E. Tarjan. 1992. Randomized parallel algorithms for trapezoidal diagrams. International fournal of Computational Geometry and Application 2 (1992), 117-133.
[12] K. Clarkson, S. Kapoor, and P. Vaidya. 1987. Rectilinear shortest paths through polygonal obstacles in $O\left(n \log ^{2} n\right)$ time. In Proceedings of the 3rd Annual Symposium on Computational Geometry (SoCG). 251-257.

[13] K. Clarkson, S. Kapoor, and P. Vaidya. 1988. Rectilinear shortest paths through polygonal obstacles in $O\left(n \log ^{2 / 3} n\right)$ time. Manuscript.

[14] P.J. de Rezende, D.T. Lee, and Y.F. Wu. 1989. Rectilinear shortest paths in the presence of rectangular barriers. Discrete and Computational Geometry 4 (1989), $41-53$.

[15] E.D. Demaine, J.S.B. Mitchell, and J. O'Rourke. 2020. The open problem project. https://topp.openproblem.net/.

[16] H. Edelsbrunner, L. Guibas, and J. Stolfi. 1986. Optimal point location in a monotone subdivision. SIAM f. Comput. 15, 2 (1986), 317-340.

[17] S.D. Eriksson-Bique, J. Hershberger, V. Polishchuk, B. Speckmann, S. Suri, T. Talvitie, K. Verbeek, and H. Ylldı. 2015. Geometric $k$ shortest paths. In Proceedings of the Twenty-Sixth Annual ACM-SIAM Symposium on Discrete Algorithms (SODA). $1616-1625$.

[18] S.K. Ghosh and D.M. Mount. 1991. An output-sensitive algorithm for computing visibility graphs. SIAM F. Comput. 20, 5 (1991), 888-910.

[19] L.J. Guibas and J. Hershberger. 1989. Optimal shortest path queries in a simple polygon. 7. Comput. System Sci. 39, 2 (1989), 126-152.

[20] L.J. Guibas, J. Hershberger, D. Leven, M. Sharir, and R.E. Tarjan. 1987. Linear-time algorithms for visibility and shortest path problems inside triangulated simple polygons. Algorithmica 2, 1-4 (1987), 209-233.

[21] J. Hershberger. 1991. A new data structure for shortest path queries in a simple polygon. Inform. Process. Lett. 38, 5 (1991), 231-235.

[22] J. Hershberger and J. Snoeyink. 1994. Computing minimum length paths of a given homotopy class. Computational Geometry: Theory and Applications 4 (1994), 63-97.

[23] J. Hershberger and S. Suri. 1999. An optimal algorithm for Euclidean shortest paths in the plane. SIAM F. Comput. 28 (1999), 2215-2256.

[24] J. Hershberger, S. Suri, and H. Yıldız. 2013. A near-optimal algorithm for shortest paths among curved obstacles in the plane. In Proceedings of the 29th Annual Symposium on Computational Geometry (SoCG). 359-368.

[25] S. Hertel and K. Mehlhorn. 1985. Fast triangulation of the plane with respect to simple polygons. Information and Control 64 (1985), 52-76.

[26] R. Inkulu, S. Kapoor, and S.N. Maheshwari. 2010. A near optimal algorithm for finding Euclidean shortest path in polygonal domain. In arXiv:1011.6481v1.

[27] S. Kapoor and S.N. Maheshwari. 1988. Efficient algorithms for Euclidean shortest path and visibility problems with polygonal obstacles. In Proceedings of 4th Annual ACM Symposium on Computational Geometry (SoCG). 172-182.

[28] S. Kapoor and S.N. Maheshwari. 2000. Efficiently constructing the visibility graph of a simple polygon with obstacles. SIAM 7. Comput. 30, 3 (2000), 847-871.

[29] S. Kapoor, S.N. Maheshwari, and J.S.B. Mitchell. 1997. An efficient algorithm for Euclidean shortest paths among polygonal obstacles in the plane. Discrete and Computational Geometry 18 (1997), 377-383.

[30] D. Kirkpatrick. 1983. Optimal search in planar subdivisions. SIAM f. Comput. 12 (1983), 28-35.

[31] D. Kirkpatrick and J. Snoeyink. 1995. Tentative prune-and-search for computing fixed-points with applications to geometric computation. Fundamenta Informaticae 22 (1995), 353-370.

[32] D.T. Lee and F.P. Preparata. 1984. Euclidean shortest paths in the presence of rectilinear barriers. Networks 14 (1984), 393-410.

[33] J.S.B. Mitchell. 1989. An optimal algorithm for shortest rectilinear paths among obstacles. Abstracts of the 1st Canadian Conference on Computational Geometry.

[34] J.S.B. Mitchell. 1991. A new algorithm for shortest paths among obstacles in the plane. Annals of Mathematics and Artificial Intelligence 3 (1991), 83-105.

[35] J.S.B. Mitchell. 1992. $L_{1}$ shortest paths among polygonal obstacles in the plane. Algorithmica 8 (1992), 55-88.

[36] J.S.B. Mitchell. 1996. Shortest paths among obstacles in the plane. International fournal of Computational Geometry and Applications 6 (1996), 309-332.

[37] J.S.B. Mitchell and S. Suri. 1995. Separation and approximation of polyhedral objects. Computational Geometry: Theory and Applications 5 (1995), 95-114.

[38] M. Overmars and J. van Leeuwen. 1981. Maintenance of configurations in the plane. 7. Comput. System Sci. 23 (1981), 166-204.

[39] H. Rohnert. 1986. Shortest paths in the plane with convex polygonal obstacles. Inform. Process. Lett. 23 (1986), 71-76.

[40] N. Sarnak and R.E. Tarjan. 1986. Planar point location using persistent search trees. Commun. ACM 29 (1986), 669-679.

[41] M. Sharir and A. Schorr. 1986. On shortest paths in polyhedral spaces. SIAM $\mathcal{F}$. Comput. 15 (1986), 193-215.

[42] J.A. Storer and J.H. Reif. 1994. Shortest paths in the plane with polygonal obstacles. 7. ACM 41 (1994), 982-1012.

[43] H. Wang. 2020. A divide-and-conquer algorithm for two-point $L_{1}$ shortest path queries in polygonal domains. Fournal of Computational Geometry 11 (2020), 235-282.

[44] H. Wang. 2021. Shortest paths among obstacles in the plane revisited. In Proceedings of the 32nd Annual Symposium on Discrete Algorithms (SODA). 810-821. 